\title{
SOCIAL NORMS, RATIONAL CHOICE AND BELIEF CHANGE
}

\author{
HORACIO ARLÓ-COSTA AND ARTHUR PAUL PEDERSEN
}

\begin{abstract}
This article elaborates on foundational issues in the social sciences and their impact on the contemporary theory of belief revision. Recent work in the foundations of economics has focused on the role external social norms play in choice. Amartya Sen has argued in [Sen93] that the traditional rationalizability approach used in the theory of rational choice has serious problems accommodating the role of social norms. Sen's more recent work [Sen96, Sen97] proposes how one might represent social norms in the theory of choice, and in a very recent article [BS07] Walter Bossert and Kotaro Suzumura develop Sen's proposal, offering an extension of the classical theory of choice that is capable of dealing with social norms.

The first part of this article offers an alternative functional characterization of the extended notion of rationality employed by Bossert and Suzumura in [BS07]. This characterization, unlike the one offered in [BS07], represents a norm-sensitive notion of rationality in terms of a pure functional constraint unmediated by a notion of revealed preference (something that is crucial for the application developed in the second part of this article). This functional characterization is formulated for general domains (as is Bossert and Suzumura's characterization) and is therefore empirically more applicable than usual characterizations of rationality. Interestingly, the functional constraint we propose is a variant of a condition first entertained in [AGM85] by Carlos Alchourrón, Peter Gärdenfors and David Makinson in the area of belief change.

The second part of this article applies the theory developed in the first part to the realm of belief change. We first point out that social norms can be invoked to concoct counterexamples against some postulates of belief change (like postulate $(* 7)$ ) that are necessary for belief change to be relational. These examples constitute the epistemological counterpart of Sen's counterexamples against condition $\alpha$ in rational choice (as a matter of fact, Rott has showed in [Rot01] that condition $\alpha$ and postulate $(* 7)$ are mutually mappable). These examples are variants of examples Rott has recently presented in [Rot04]. One of our main goals in this article consists in applying the theory developed in the first part to develop a theory of norm-inclusive belief change that circumvents the counterexamples. We offer a new axiomatization for belief change and we furnish correspondence results relating constraints of rational choice to postulates of belief change.
\end{abstract}

\section{INTRODUCTION}

The classical theory of rational choice as developed by mathematical economists such as Kenneth Arrow [Arr51, Arr59], Marcel K. Richter [Ric66, Ric71], Paul Samuelson [Sam38, Sam47], and Amartya Sen [Sen70, Sen71] has occupied a central role in the philosophy of the social sciences for almost a century now. Jon Elster has provided a convincing argument for the applicability of the theory to the social sciences in various books and articles (see, for example, [Els89a]). The theory also occupies a central foundational role in the contemporary theory of belief change.

We wish to thank Isaac Levi, David Makinson, and Teddy Seidenfeld for valuable comments on earlier versions of this article. 
In fact, recently, Hans Rott has shown how so-called rationality postulates of belief change and non-monotonic reasoning correspond in a one-to-one fashion to so-called coherence constraints of classical theories of rational choice [Rot01]. In particular, Rott provides a connection between classical coherence constraints on selection functions of rational choice and rationality constraints on operators of belief change and non-monotonic reasoning. A recurrent feature of formal theories of belief change and non-monotonic reasoning is that some extralogical selection function is employed to do the dirty work, and Rott's study rigorously exhibits how this feature affords a nexus between formal theories of belief change and non-monotonic reasoning. ${ }^{1}$ But more importantly, Rott's work shows that belief change and non-monotonic reasoning can be investigated in the formal framework of rational choice. ${ }^{2}$

Amartya Sen, who contributed crucially to the development of the received view of rational choice, has also been one of its main critics. In fact, in a series of articles [Sen93, Sen96, Sen97] Sen has argued convincingly against the a priori imposition of requirements of internal consistency of choice, such as the weak and the strong axioms of revealed preference, Arrow's axiom of choice consistency, and Sen's Property $\alpha$. The following example, presented in [Sen93], can give the reader an idea of the difficulties that Sen has in mind:

Suppose...[a] person faces a choice at a dinner table between having the last remaining apple in the fruit basket $(y)$ and having nothing instead $(x)$, forgoing the nice-looking apple. She decides to behave decently and picks nothing $(x)$, rather than the one apple $(y)$. If, instead, the basket had contained two apples, and she had encountered the choice between having nothing $(x)$, having one nice apple $(y)$ and having another nice one $(z)$, she could reasonably enough choose one $(y)$, without violating any rule of good behavior. The presence of another apple $(z)$ makes one of the two apples decently choosable, but this combination of choices would violate the standard consistency conditions, including Property $\alpha$, even though there is nothing particularly "inconsistent" in this pair of choices ... (p. 501).

This example, as many others offered by Sen, involves a social norm, in this case a rule of politeness that seems to make the option $y$ unavailable for the person when she is faced with the first choice. As Sen indicates, the combination of choices the person takes in this example violates standard consistency conditions (also known as coherence constraints) such as condition $\alpha$, which demands that whatever is rejected for choice must remain rejected if the set of alternatives available for choice is expanded. Examples like this one

\footnotetext{
${ }^{1}$ The relationship between formal theories of belief change and non-monotonic reasoning has been well examined in [GM94] and [MG91]. For arguments calling into question the claim that the AGM theory of belief change and the Rational Logic of Kraus, Lehmann and Magidor [KLM90] are two sides of the same coin, see [Ar195].

${ }^{2}$ Occasionally Rott claims in addition that his formal results should be interpreted as a formal reduction of theoretical rationality to practical rationality or, less ambitiously, as a way of utilizing the theory of choice functions as a more primitive (and secure) theory to which the theory of belief revision has been reduced (e.g., see [Rot01, pp. 5-6, 142, 214]). It is unclear whether these claims hold independently of the formal results presented by Rott. In [Ols03], Erik Olsson offers criticisms along these lines. Isaac Levi argues in [Lev04b] that the reduction is not a reduction to a theory of choice per se. In addition, Levi offers in [Lev04b] an analysis of belief change where the act of changing view is constructed as an epistemic decision. The main technical tools Levi uses are not taken from the theory of choice functions but from other areas of decision theory.

We will appeal to some of the techniques Rott uses in [Rot01], but we do not claim that we are offering a reduction of belief change to the theory of choice or a reduction of theoretical rationality to practical rationality. As the reader will see, nevertheless, the mathematical techniques Rott exploits in [Rot01] have an interesting heuristic value to discover interesting postulates regulating belief change when social norms are relevant.
} 
have led many to believe that the standard rationalizability approach to the theory of choice faces serious difficulties coping with the existence of external social norms. ${ }^{3}$

The above example, where social norms influence choice, is an illustration of a phenomenon that Sen calls menu dependence. Roughly speaking, menu dependence arises in rational choice when the evaluation of alternatives for choice or the mode of selection guiding choice varies parametrically with what collection of alternatives is available for choice. For instance, menu dependence may arise when a set of alternatives-often called a menu in rational choice-from which an agent is to choose carries information directly relevant to what the agent has reasons to choose. In particular, an agent faced with choosing among some set of alternatives may learn something about the underlying situation, thereby influencing the agent's decision over the alternatives available for choice.

To take an example, a chooser may learn something about a person offering a choice on the basis of what the person is offering: Given the choice between having a beer after work at a distant acquaintance's home $(b)$ and not spending time with the acquaintance $(s)$, a person who chooses to have a beer instead of going home may nevertheless choose to not spend time with the acquaintance if the acquaintance instead offers a choice among having a beer $(b)$, not spending time with the acquaintance $(s)$, and smoking some crack (c). The appearance of the third alternative has altered the person's evaluation of the other two alternatives. In particular, the person has learned something about the acquaintance when offered the third alternative, and so the expanded menu has triggered additional inferences about the acquaintance; accordingly, the person chooses to not spend time with the acquaintance. In this example, the set of alternatives $\{b, s, c\}$ has epistemic relevance for the person's decision, and the person's choices are menu dependent. The foregoing example is also illustration of a violation of condition $\alpha$, since $s$ was rejected for choice from $\{b, s\}$ yet was chosen from $\{b, s, c\}$.

Examples in which social norms influence choice (like the first example involving the fruit basket) can also be seen to constitute a case of menu dependence of choice. In the case of the fruit basket, the menu $\{x, y\}$ occasions a particular mode of choice, i.e., a particular means by which choice is made. In this case there is a concrete mechanism that explains why this is the case. Option $y$ is rendered unfeasible for choice from the menu $\{x, y\}$ in virtue of an operative social norm, whereupon the person chooses $x$ according to her preferences. When $z$ is added to the menu, the norm is no longer applicable, whereby $y$ becomes available for choice for the person and she chooses $y$ according to her preferences. We find especially interesting and more tractable cases in which social norms place constraints upon maximizing according to fixed preferences. We will accordingly focus on cases of this sort in this article.

In light of Rott's correspondence results, it is natural to inquire whether violations of rationality postulates of belief change and non-monotonic reasoning can be understood as a result of menu dependence and in particular operative social norms. In other words, it is natural to ask whether menu dependence, which can wreak havoc on almost all coherence constraints on selection functions in rational choice, can-in epistemic form-also undercut rationality postulates of belief change and non-monotonic reasoning. We will see that

\footnotetext{
${ }^{3}$ There are many possible responses to Sen's examples. One option could be to redefine the space of options in such a way as to tag $y$ as the option of 'taking the last apple from the plate' (see [Lev04a] for an analysis along these lines). Sen himself seems ambivalent regarding the analysis of his own examples. On some occasions he seems to think that redefinitions of this sort are feasible, yet on other occasions he has argued that these type of redefinitions make the principles of rational choice empty. In general, maneuvers of this kind tend to be blind with respect to the role of social norms in reasoning. We prefer here to take norms at face value as Sen does in many of his writings.
} 
some of the existing counterexamples against well-known principles of belief change (of the sort proposed by Rott in [Rot04]) are indeed explicable in terms of the phenomenon of menu dependence. In addition, we present counterexamples for which menu dependence is explainable in terms of social norms.

The methodological difficulty that the possibility of menu dependence and in particular social norms pose for belief change and non-monotonic reasoning is by no means trivial. Indeed, as we will see, the possibility of menu dependence threatens an all but universal presumption in the literature of belief change and non-monotonic reasoning-that selection functions are relational. ${ }^{4}$ Roughly, what this presumption amounts to is the requirement that there is some underlying binary relation-whether over all sentences, sets of sentences, worlds, sets of worlds, models, or sets of models, and so on-according to which a selection function picks the 'best' or 'unsurpassed' elements from its arguments. As discussed by Sen [Sen97], social norms (and menu dependence in general) threatens essentially the same presumption in the context of rational choice-that selections function are rationalizable. This presumption requires that there is some underlying binary preference relation over all alternatives for choice according to which a selection function picks optimal or maximal elements from its arguments. In fact, relationality in belief change and non-monotonic reasoning is formally equivalent to rationalizability in rational choice. ${ }^{5}$

Some of the recent literature [BS07] has argued that it is possible to accommodate social norms in the theory of rational choice by adopting suitably modified axioms of revealed preference. In this article we offer an argument along these lines that improves on the existing arguments presented in [BS07]. We will use this result to provide a solution to some of the problems previously diagnosed in the theory of belief change, offering a novel axiomatization of belief change that, we claim, helps to resolve problems recently raised against standard axiomatizations of belief revision à la Alchourrón, Gärdenfors, and Makinson [AGM85] (AGM). Surprisingly perhaps (taking into account that we arrive at our conditions by way of a completely different route), the central condition deployed in our improved argument is a variant of a condition entertained by AGM in [AGM85]. So in a way the main new axioms we propose have precursors in the literature.

Our article intends to show that the debate regarding the foundations of the theory of rational choice is directly relevant to the understanding of apparent counterexamples against principles of belief formation used in the theory of belief change (for example, the counterexamples presented in $[\operatorname{Rot} 04])$. At the same time we intend to contribute to the contemporary debate in the foundations of the social sciences itself by showing the robustness of the program of rationalizability both in rational choice and in belief change. Thus, we intend to offer a common solution to problems that many have identified as fatal for the foundational program defended by Rott (see, for example, [Ols03]) and for the rationalizability program in the foundations of rational choice.

The article is divided into two halves, each of which has two parts. Part I is structured as follows. In Section 1.1, we present the technical machinery used both in the half devoted to rational choice and in the half devoted to belief revision. Section 1.2 offers an introduction to the theory of choice functions in rational choice. We review the traditional notion of rationalizability in terms of optimization and several coherence constraints on choices across varying menus that have been important in the literature. Then we propose a condition in

\footnotetext{
${ }^{4} \mathrm{~A}$ non-exhaustive list of influential articles in belief change and non-monotonic reasoning which presume relationality: [AGM85], [AM85], [Rot93], [RP00], [Han99], [Ar106]; [Mak89], [KLM90], [LM92], [GM94].

${ }^{5}$ In fact, the influence of social norms and more generally menu dependence even threatens the presumption that selection functions are pseudo-rationalizable (see [Mou85] for a discussion of this notion).
} 
terms of which it is possible to formulate a functional characterization of rationalizability with respect to general domains. We will see that this condition is a natural generalization of a condition first entertained in the seminal paper by AGM on belief change [AGM85].

In Part II, we first focus in Section 2.1 on attempts to extend the notion of rationalizability to cope with social norms. We discuss the work of Walter Bossert and Kotaro Suzumura [BS07] and present their extension of the theory of rationalizability. Section 2.2 presents an alternative extension of rationalizability capable of accommodating social norms that we call norm-conditional choice. We compare our extension with Bossert and Suzumura's extension. We argue that our proposal offers some advantages that will play a crucial role in the sections on epistemological applications that follow.

Part III initiates the second half of the article. In Section 3.1 we present basic background on the literature of belief revision. Then in Section 3.2 the use of selection functions in belief revision is connected formally with the use of choice functions in rational choice. Section 3.3 is a self-contained review of some of the central results offered by Rott in [Rot01] linking postulates of belief revision to coherence constraints in the theory of rational choice.

Section 3.4 presents various counterexamples to principles of belief formation necessary for rationalizability, such as postulate $(* 7)$. The initial counterexample is due to Rott [Rot04]. Then we present new examples that illustrate the role of social norms in belief revision.

In Part IV, Section 4.1 presents a new theory of belief revision called norm-inclusive belief revision. Like the theory of norm-conditional choice we discuss, norm-inclusive belief revision is intended to take into account the role social norms play in belief change. In Section 5 we discuss our theory and illustrate how our theory works. Section 6 closes the paper with a conclusion and a discussion of future work.

\section{PART I: New foundations for Rational Choice}

1.1. Technical Preliminaries. In the following we presuppose a propositional language $\mathcal{L}$ with the connectives $\neg, \wedge, \vee, \rightarrow, \leftrightarrow$. We let $\operatorname{For}(\mathcal{L})$ denote the set of formulae of $\mathcal{L}$, $a, b, c, \ldots p, q, r, \ldots$ denote propositional variables of $\mathcal{L}$, and $\alpha, \beta, \delta, \ldots, \varphi, \psi, \chi, \ldots$ denote arbitrary formulae of $\mathcal{L}$. Sometimes we assume that the underlying language $\mathcal{L}$ is finite. By this we mean that $\mathcal{L}$ has only finitely many propositional variables.

As is customary, we assume that $\mathcal{L}$ is governed by a consequence operation $\mathrm{Cn}$ : $\mathscr{P}(\operatorname{For}(\mathcal{L})) \rightarrow \mathscr{P}(\operatorname{For}(\mathcal{L}))$ such that for all $A, B \subseteq \operatorname{For}(\mathcal{L})$,

(i) $A \subseteq \mathrm{Cn}(A)$.

(ii) If $A \subseteq B$, then $\operatorname{Cn}(A) \subseteq \operatorname{Cn}(B)$.

(iii) $\mathrm{Cn}(\mathrm{Cn}(A)) \subseteq \operatorname{Cn}(A)$.

(iv) $\mathrm{Cn}_{0}(A) \subseteq \mathrm{Cn}(A)$, where $\mathrm{Cn}_{0}$ is classical tautological implication.

(v) If $\varphi \in \operatorname{Cn}(A)$, then there is some finite $A_{0} \subseteq A$ such that $\varphi \in \operatorname{Cn}\left(A_{0}\right)$.

(vi) If $\varphi \in \operatorname{Cn}(A \cup\{\psi\})$, then $\psi \rightarrow \varphi \in \operatorname{Cn}(A)$.

Conditions (i)-(vi) are respectively called Inclusion, Monotony, Idempotence, Supraclassicality, Compactness, and Deduction [Han99, p. 26]. As usual, $A$ is called logically closed if $\operatorname{Cn}(A)=A$, and $A \vdash \varphi$ is an abbreviation for $\varphi \in \operatorname{Cn}(A)$. We let $\mathbb{K}$ denote the collection of logically closed sets in $\mathcal{L}$.

We let $\mathcal{W}_{\mathcal{L}}$ denote the collection of all maximal consistent sets of $\mathcal{L}$ with respect to $\mathrm{Cn}$. Members of $\mathcal{W}_{\mathcal{L}}$ are often called states, possible worlds or just worlds. For a non-empty collection of worlds $W$ of $\mathcal{W}_{\mathcal{L}}$, let $\widehat{W}$ denote the set of sentences of $\mathcal{L}$ which are members of all worlds in $W$ (briefly, $\widehat{W}:=\bigcap_{w \in W} w$ ). If $A$ is a set of sentences of $\mathcal{L}$, we let $\llbracket A \rrbracket:=$ 
$\left\{w \in \mathcal{W}_{\mathcal{L}}: A \subseteq w\right\}$. If $\varphi$ is a sentence of $\mathcal{L}$, we write $\llbracket \varphi \rrbracket$ instead of $\llbracket\{\varphi\} \rrbracket$. Observe that for every set of sentences $A$ of $\mathcal{L}, \operatorname{Cn}(A)=\widehat{\llbracket A \rrbracket}$. A member of $\mathscr{P}\left(\mathcal{W}_{\mathcal{L}}\right)$ is often called a proposition, and $\llbracket \varphi \rrbracket$ is often called the proposition expressed by $\varphi$. Intuitively, $\llbracket A \rrbracket$ consists of those worlds in which all sentences in $A$ hold. Finally, let $\mathcal{E}_{\mathcal{L}}$ be the set of all elementary subsets of $\mathcal{W}_{\mathcal{L}}$, i.e., $\mathcal{E}_{\mathcal{L}}:=\left\{W \in \mathscr{P}\left(\mathcal{W}_{\mathcal{L}}\right): W=\llbracket \varphi \rrbracket\right.$ for some $\left.\varphi \in \operatorname{For}(\mathcal{L})\right\}$.

We now briefly turn to selection functions. For a non-empty set $X$ and a non-empty collection $\mathscr{S}$ of subsets of $X$, we call the pair $(X, \mathscr{S})$ a choice space. A selection function (or choice function) on a choice space $(X, \mathscr{S})$ is a function $\gamma: \mathscr{S} \rightarrow \mathscr{P}(X)$ such that $\gamma(S) \subseteq S$ for every $S \in \mathscr{S}^{6}$ Intuitively, a selection function $\gamma: \mathscr{S} \rightarrow \mathscr{P}(X)$ chooses the 'best' elements of each $S$ in $\mathscr{S}$.

Now let $(X, \mathscr{S})$ be a choice space. We say that $\mathscr{S}$ is closed under finite unions if for every $n<\omega$, if $\left\{S_{i}: i<n\right\} \subseteq \mathscr{S}$, then $\bigcup_{i<n} S_{i} \in \mathscr{S}$; we say that $\mathscr{S}$ is closed under relative complements if whenever $S, T \in \mathscr{S}, S \backslash T \in \mathscr{S}$; and we call $\mathscr{S}$ compact if for every $S \in \mathscr{S}$ and $I \subseteq \mathscr{S}$, if $S \subseteq \bigcup_{T \in I} T$, then there is some finite $I_{0} \subseteq I$ such that $S \subseteq \bigcup_{T \in I_{0}} T$. We also say that $(X, \mathscr{S})$ is closed under finite unions (closed under relative complements, compact) if $\mathscr{S}$ is closed under finite unions (closed under relative complements, compact).

1.2. Choice Functions in Rational Choice. The idea that a selection function $\gamma: \mathscr{S} \rightarrow$ $\mathscr{P}(X)$ takes the 'best' elements of each set in $\mathscr{S}$ has been made more precise by assuming that there is some ordering over the elements of $X$ according to which $\gamma(S)$ distinguishes the best elements of each $S \in \mathscr{S}$. Two formalizations of this idea have been widely utilized in the literature. The first formalization is based on a non-strict (reflexive) relation $\geq$ on $X$. It demands that for all $S \in \mathscr{S}$,

$\left(\mathrm{Eq}_{\geq}\right) \quad \gamma(S)=\{x \in S: x \geq y$ for all $y \in S\}$.

This formalization is called optimization in [Sen97, p. 763] and G-rationality in [Suz83, p. 21]. The second formalization of the idea of picking the 'best' elements is based on a strict (asymmetric) relation $>$ on $X$. It demands that for all $S \in \mathscr{S}$,

$\left(\mathrm{Eq}_{>}\right) \quad \gamma(S)=\{x \in S: y>x$ for no $y \in S\}$.

This is called maximization in [Sen97, p. 763] and M-rationality in [Suz83, p. 21]. This formalization captures a somewhat weaker notion than that of picking the 'best' elements of each $S \in \mathscr{S}$. Indeed, it would be more accurate to say $\mathrm{Eq}_{>}$is a formalization of the idea of picking elements that are 'no worse' than any other elements. In this article, much of our discussion concerning rational choice will be framed with respect to optimization.

In rational choice, the ideas represented in $\mathrm{Eq}_{\geq}$and $\mathrm{Eq}_{>}$have been quite generally exploited, the methodology of rational choice characterized quite well by the slogan, 'Rational choice is relational choice' [Rot93, p. 1429]. There the elements of $X$ from a choice space $(X, \mathscr{S})$ represent alternatives of choice under the potential control of an agent, the members of $\mathscr{S}$ represent possible decision problems for the agent, and a selection function $\gamma$ on $(X, \mathscr{S})$ represents the values of the agent. Then for an agent confronted with a decision problem $S$ (also often called a menu), the set $\gamma(S)$ consists of those elements that the agent regards as equally adequate or satisfactory alternatives from $S[\mathrm{Her} 73$, p. 189]. ${ }^{7}$ Accordingly, choice is often construed as that which is based upon an underlying

\footnotetext{
${ }^{6}$ Following Rott [Rot01], we do not require that $\mathscr{S}$ or $\gamma(\mathscr{S})$ consists solely of nonempty sets. This approach allows for more generality.

${ }^{7}$ Herzberger also writes, "Under the natural interpretation of $\gamma(S)$ as a set of solutions to the problem $S$, the value $\gamma(S)=\emptyset$ earmarks a decision problem that is unsolvable by the function $\gamma$; and so the domain $\mathscr{S}$ bears
} 
preference relation, where $\gamma(S)$, often called a choice set, represents those alternatives an agent takes to be 'best' or 'no worse' with respect to his underlying preferences.

Before we continue, let us recapitulate the forgoing discussion with a few defintions.

Definition 1.1. Let $\gamma$ be a selection function on a choice space $(X, \mathscr{S})$. We say that a binary relation $R$ on $X$ rationalizes $\gamma$ if for every $S \in \mathscr{S}$,

$$
\gamma(S)=\{x \in S: x R y \text { for all } y \in S\} .
$$

We thereby call $\gamma$ rational (or rationalizable) if there is a binary relation $R$ on $X$ that rationalizes $\gamma^{8}$

Definition 1.2. Let $\gamma$ be a selection function on a choice space $(X, \mathscr{S})$.

(i) We say that $\gamma$ is $G$-rational (or $G$-rationalizable) if there is a reflexive binary relation $\geq$ on $X$ that rationalizes $\gamma$.

(ii) We say that $\gamma$ is $M$-rational (or $M$-rationalizable) if there is an asymmetric binary relation $>$ on $X$ such that $((X \times X) \backslash>)^{-1}$ rationalizes $\gamma{ }^{9}$

Clearly a selection function $\gamma$ on a choice space $(X, \mathscr{S})$ is G-rational just in case there is a reflexive binary relation $\geq$ on $X$ such that $\gamma$ satisfies $\mathrm{Eq}_{>}$for every $S \in \mathscr{S}$. Moreover, a selection function $\gamma$ on $(X, \mathscr{S})$ is M-rational if and only if there is a asymmetric binary relation $>$ on $X$ such that $\gamma$ satisfies $\mathrm{Eq}_{>}$for every $S \in \mathscr{S}$. Thus, G-rational and Mrational selection functions correspond to the notions discussed above. In addition, every M-rational selection function is G-rational, but the converse does not in general hold. ${ }^{10}$ It is in this way that maximization is a weaker notion than optimization.

In the study of rational choice, coherence constraints have been imposed on the form relationships may take among choices across varying menus. In other words, these requirements specify how choices must be made across different decision problems. Some predominant coherence constraints are the following: ${ }^{11}$

$(\alpha)$ For every $S, T \in \mathscr{S}$, if $S \subseteq T$, then $S \cap \gamma(T) \subseteq \gamma(S)$. (Sen's Property $\alpha$ )

$\left(\beta^{+}\right) \quad$ For every $S, T \in \mathscr{S}$, if $S \subseteq T$ and $S \cap \gamma(T) \neq \emptyset$, then $\gamma(S) \subseteq \gamma(T)$. (Sen's Property $\beta^{+}$)

$(\gamma)$ For every nonempty $I \subseteq \mathscr{S}$ such that $\bigcup_{S \in I} S \in \mathscr{S}$, (Sen's Property $\gamma$ ) $\bigcap_{S \in I} \gamma(S) \subseteq \gamma\left(\bigcup_{S \in I} S\right)$.

(Aiz) For every $S, T \in \mathscr{S}$, if $S \subseteq T$ and $\gamma(T) \subseteq S$, then $\gamma(S) \subseteq \gamma(T)$.

(Aizerman's Axiom)

interpretation as the class of all decision problems that are solvable under the given choice function" [Her73, p. 189 , notation adapted].

${ }^{8}$ There are alternative notions of rationalizablity one might find appealing. For example, we might instead say that a binary relation $R$ on $X$ rationalizes $\gamma$ if for every $S \in \mathscr{S}$ such that $\gamma(S) \neq \emptyset, \gamma(S)=\{x \in S$ : $x R y$ for all $y \in S\}$.

${ }^{9}$ For a binary relation $R$ on $X, R^{-1}:=\{(x, y) \in X \times X:(y, x) \in R\}$.

${ }^{10}$ However, every selection function rationalized by a complete binary relation is M-rational. (A binary relation $R$ on $X$ is complete if for every $x, y \in X$, either $x R y$ or $y R x$.) To see why not every G-rational selection function is M-rational, we present an example from [Suz76, pp. 151-152].

Consider a selection function $\gamma$ on a choice space $(X, \mathscr{S})$, where $X:=\{x, y, z\}, \mathscr{S}:=$ $\{\{x, y\},\{x, z\}, X\}, \gamma(\{x, y\}):=\{x, y\}, \gamma(\{x, z\}):=\{x, z\}$, and $\gamma(\{x, y, z\}):=\{x\}$. Then $\gamma$ is rationalized by a reflexive binary relation $\geq$ defined by $\geq:=\{(x, x),(y, y),(z, z),(x, y),(x, z),(y, x),(z, x)\}$. However, $\gamma$ is not M-rationalizable, for otherwise, if $>$ is an asymmetric binary relation for which $(X \backslash>)^{-1}$ rationalizes $\gamma$, then since $\gamma(\{x, y\})=\{x, y\}$ and $\gamma(\{x, z\})=\{x, z\}$, it follows that $x \ngtr y$ and $x \ngtr z$, but since $\gamma(\{x, y, z\})=\{x\}$, it follows that $y>z$ and $z>y$, contradicting that $>$ is asymmetric.

${ }^{11}$ Actually, Sen's Property $\beta$ is more pervasive than Sen's Property $\beta^{+}$[Sen77, p. 66]. Condition $\beta$ demands that if $S \subseteq S^{\prime}$ and $\gamma\left(S^{\prime}\right) \cap \gamma(S) \neq \emptyset$, then $\gamma(S) \subseteq \gamma\left(S^{\prime}\right)$ [Sen71, p. 313]. Condition $\beta^{+}$entails condition $\beta$, and in the presence of condition $\alpha$, condition $\beta$ and condition $\beta^{+}$are logically equivalent. 
We mentioned condition $\alpha$ in the introduction of this article. Recall that this condition demands that whatever is rejected for choice from a menu must remain rejected if the menu is expanded. More formally, this means that for any menu $S$, if $x$ is an alternative in $S$ and $x$ is not in $\gamma(S)$-that is, $x$ is not chosen, i.e., is rejected, from $S$-then if $S$ is expanded to a menu $S^{\prime}$ - that is, if $S^{\prime}$ is such that $S$ is a subset of $S^{\prime}$-then $x$ is not in $\gamma\left(S^{\prime}\right)$. Equivalently, this condition demands that whatever is chosen from a menu must also be chosen from any smaller menu for which this choice is still available. ${ }^{12}$ Condition $\alpha$ entails the following coherence constraint:

$\left(\alpha^{*}\right) \quad$ For every $S, T \in \mathscr{S}$ such that $S \cup T \in \mathscr{S}, \gamma(S \cup T) \subseteq \gamma(S) \cup \gamma(T)$. (Sen's Property $\alpha^{*}$ )

Furthermore, if $\mathscr{S}$ is closed under finite unions and relative complements, then condition $\alpha$ is equivalent to condition $\alpha^{*}$.

Similarly, condition $\gamma$ entails the following coherence constraint:

$\left(\gamma^{*}\right) \quad$ For every $S, T \in \mathscr{S}$ such that $S \cup T \in \mathscr{S}, \gamma(S) \cap \gamma(T) \subseteq \gamma(S \cup T)$. (Sen's Property $\gamma^{*}$ )

If $\mathscr{S}$ is closed under finite unions and is compact, then condition $\gamma$ is equivalent to condition $\gamma^{*}$.

We can combine $\alpha^{*}$ and $\gamma^{*}$ into one condition:

$(\gamma \mathrm{R})$ For every $S, T \in \mathscr{S}$ such that $S \cup T \in \mathscr{S}$, $\gamma(S) \cap \gamma(T) \subseteq \gamma(S \cup T) \subseteq \gamma(S) \cup \gamma(T)$.

Now observe that if $\mathscr{S}$ is closed under finite unions and is compact, the following coherence constraints are pairwise equivalent:

$$
\begin{aligned}
&\left(\gamma \mathrm{R}_{\infty}\right) \quad \text { For every nonempty } I \subseteq \mathscr{S} \text { and } S \in \mathscr{S}, \\
& \text { if } S \subseteq \bigcup_{T \in I} T, \text { then } S \cap\left(\bigcap_{T \in I} \gamma(T)\right) \subseteq \gamma(S) . \\
&\left(\gamma \mathrm{R}_{<\omega}\right) \quad \text { For every } n<\omega, \text { if } T_{i} \in \mathscr{S} \text { for each } i<n \text { and } S \in \mathscr{S}, \text { then } \\
& \text { if } S \subseteq \bigcup_{i<n} T_{i} \text {, then } S \cap\left(\bigcap_{i<n} \gamma(T)\right) \subseteq \gamma(S) . \\
&\left(\gamma \mathrm{R}_{1}\right) \quad \text { For every } S, T_{0}, T_{1} \in \mathscr{S} \\
& \text { if } S \subseteq T_{0} \cup T_{1}, \text { then } S \cap \gamma\left(T_{0}\right) \cap \gamma\left(T_{1}\right) \subseteq \gamma(S) .
\end{aligned}
$$

If in addition $\mathscr{S}$ is closed under relative complements, then condition $\gamma \mathrm{R}$ is equivalent to each of the aforementioned coherence constraints. We record this fact in a proposition, leaving its proof to the reader.

Proposition 1.3. Let $\gamma$ be a selection function on a choice space $(X, \mathscr{S})$ that is closed under finite unions and is compact. Then the following are equivalent:

(i) $\gamma$ satisfies $\gamma \mathrm{R}_{\infty}$.

(ii) $\gamma$ satisfies $\gamma \mathrm{R}_{<\omega}$.

(iii) $\gamma$ satisfies $\gamma \mathbf{R}_{1}$.

(iv) $\gamma$ satisfies $\alpha$ and $\gamma$.

(v) $\gamma$ satisfies $\alpha$ and $\gamma^{*}$.

If in addition $(X, \mathscr{S})$ is closed under relative complements, then the following are pairwise equivalent and equivalent to $\gamma \mathrm{R}_{\infty}$ :

(vi) $\gamma$ satisfies $\gamma \mathrm{R}$.

(vii) $\gamma$ satisfies $\alpha^{*}$ and $\gamma$.

(viii) $\gamma$ satisfies $\alpha^{*}$ and $\gamma^{*}$.

\footnotetext{
${ }^{12}$ Condition $\alpha$, also known as Chernoff's Axiom, should not be confused with another important condition, the so-called Independence of Irrelevant Alternatives [Arr51, p. 27]. See [Sen77, pp. 78-80] for a vivid discussion of the difference between these two conditions. See also [Ray73] for another clear discussion of this sort.
} 
We invite the reader to consider the implications among the above coherence constraints (in particular, observe that condition $\gamma \mathbf{R}_{\infty}$ entails conditions $\alpha$ and $\gamma$ ).

It is well-known that conditions $\alpha, \alpha^{*}, \gamma$, and $\gamma^{*}$ are each necessary for a selection function to be rationalizable. Indeed, it can be shown that conditions $\gamma \mathbf{R}, \gamma \mathbf{R}_{\infty}, \gamma \mathbf{R}_{<\omega}$, and $\gamma \mathbf{R}_{1}$ are also each necessary for a selection function to be rationalizable.

Proposition 1.4. A rational selection function $\gamma$ satisfies $\alpha, \alpha^{*}, \gamma, \gamma^{*}, \gamma \mathbf{R}, \gamma \mathbf{R}_{\infty}, \gamma \mathbf{R}_{<\omega}$, and $\gamma \mathbf{R}_{1}$.

Yet only select subsets of these conditions are sufficient for a selection function to be rationalizable. To be sure, conditions $\alpha$ and $\gamma$ are jointly sufficient for rationalizability, as are $\alpha^{*}$ and $\gamma, \alpha$ and $\gamma^{*}$, and $\alpha^{*}$ and $\gamma^{*}$. Furthermore, conditions $\gamma \mathbf{R}, \gamma \mathbf{R}_{<\omega}, \gamma \mathbf{R}_{1}$ are each sufficient to rationalize a choice function. However, only under certain constraints on domains of selection functions are any of the aforementioned conditions sufficient. Thus, in the spirit of generality, we offer a general characterization of rationalizability which does not depend on any domain restrictions.

Theorem 1.5. A selection function $\gamma$ is rational if and only if it satisfies condition $\gamma \mathbf{R}_{\infty} .{ }^{13}$

For the sake of brevity, we omit a proof this theorem, referring the reader to Theorem 2.6 below to see how to assemble a proof of the above theorem. The reader may consult the proof in [Ric71, Theorem 2, p. 33] to gather some of the elements required for a proof of the above theorem. ${ }^{14}$

In [Ric71], as in most literature of kin, selection functions are assumed to exclude the empty set from their domains and are moreover assumed to satisfy the following condition:

$$
\left(\gamma_{>\emptyset}\right) \quad \text { For every } S \in \mathscr{S} \text {, if } S \neq \emptyset \text {, then } \gamma(S) \neq \emptyset \text {. (Regularity) }
$$

Rott calls this condition success in [Rot01, p. 150]. We will call a selection function that satisfies condition $\gamma_{>\emptyset}$ regular. In the presence of condition $\gamma_{>\emptyset}$, a selection function satisfying condition $\gamma \mathbf{R}_{\infty}$ is G-rational, i.e., rationalized by a reflexive binary relation. But one need not presuppose condition $\gamma_{>\emptyset}$ in a theorem. Here we offer a condition which in conjunction with condition $\gamma \mathbf{R}_{\infty}$ is sufficient for G-rationality:

$\left(\gamma_{1>\emptyset}\right) \quad$ For every $x \in X$ such that $\{x\} \in \mathscr{S}, \gamma(\{x\}) \neq \emptyset$. (Singleton Regularity)

We thereby have the following theorem, which we offer without proof. ${ }^{15}$

Theorem 1.6. A selection function $\gamma$ is G-rational if and only if it satisfies condition $\gamma \mathbf{R}_{\infty}$ and condition $\gamma_{1>\emptyset}$.

We immediately have the following corollary:

Corollary 1.7. Let $\gamma$ be a selection function satisfying condition $\gamma_{>\emptyset}$. Then $\gamma$ is $G$ rationalizable if and only if it satisfies condition $\gamma \mathbf{R}_{\infty}$.

\footnotetext{
${ }^{13}$ Recall the notion of rationalizability briefly discussed in footnote 8 . It can be shown that a selection function is rationalizable in the sense of footnote 8 just in case it satisfies the following condition: For every nonempty $I \subseteq \mathscr{S}$ and $S \in \mathscr{S}$, if $S \subseteq \bigcup_{T \in I} T$ and $\gamma(S) \neq \emptyset$, then $S \cap\left(\bigcap_{T \in I} \gamma(T)\right) \subseteq \gamma(S)$. This illustrates how one can modify coherence constraints for other notions of rationalizablity.

${ }^{14}$ Theorem 2.6 is stated within a more general framework that we introduce in Section 2.2. A direct proof of Theorem 1.5 proceeds in a way unlike the proof in [Ric71]. This is primarily because the results in [Ric71] concern what is called the $V$-Axiom. Condition $\gamma \mathbf{R}_{\infty}$ is not discussed in [Ric71].

${ }^{15}$ The minimal conditions needed for a proof can be gathered from the proof in [Ric71, Theorem 3, p. 34]. Although the proof in [Ric71] does not itself establish Theorem 1.6, a careful inspection of the proof in [Ric71] should make it clear that some assumptions of the theorem associated with this proof can be weakened. Indeed, condition $\gamma_{1>\emptyset}$ is not discussed in [Ric71] or to our knowledge anywhere else in the literature on choice functions. As we have indicated, selection functions are assumed to be regular in [Ric71]. See footnote 14 .
} 
The astute reader will have observed that conditions $\gamma \mathbf{R}_{\infty}, \gamma \mathbf{R}_{<\omega}$, and $\gamma \mathbf{R}_{1}$ bear a striking resemblance to conditions $\gamma 7: \infty, \gamma 7: N$, and $\gamma 7: 2$ of [AGM85]. Indeed, $\gamma \mathbf{R}_{\infty}$, $\gamma \mathbf{R}_{<\omega}$, and $\gamma \mathbf{R}_{1}$ are generalized forms of these conditions. As Rott points out in [Rot93, p. 1432], Alchourrón, Gärdenfors, and Makinson realized that conditions $\gamma 7: 2$ and $\gamma 7: \infty$ are each alone sufficient for rationalizability in the context of their theory of belief change [AGM85, p. 521-522, 529-530] (in the context of the AGM theory of contraction, the domain of each selection function is closed under finite unions, closed under relative complements, and compact). ${ }^{16}$ They posed as an open question whether condition $\gamma 7: 2$ (which is a special case of our $\gamma \mathbf{R}_{1}$ ) can be expressed as a rationality postulate of belief contraction. Rott provides an affirmative answer to this question in [Rot93], showing first that $\gamma 7: 2$ can be decomposed into conditions $\alpha^{*}$ and $\gamma^{*}$, whereupon he demonstrates that condition $\alpha^{*}$ corresponds to rationality postulate $(-7)$, while under certain assumptions condition $\gamma^{*}$ corresponds to rationality postulate $(-8 r)$. ${ }^{17}$

In the next section, we will discuss recent work in rational choice aimed at accommodating the possibility of menu dependence in the standard theory of choice. We will focus on a theory of choice that takes into account the influence of social norms in choice. Thereafter we will adopt some of the central ideas of this theory to develop a theory that we take to be an improvement upon the old one.

\section{PART II: Social Norms And Rational Choice}

2.1. Norm-Conditional Rationalizability. In [Sen93, Sen96, Sen97], Amartya Sen considers one aspect of the general phenomenon of menu dependence-namely, the problems external social norms pose for the theory of choice. Sen's examples seem to show that the standard rationalizability approach to the theory of choice-as exercised by Arrow [Arr59], Hansson [Han68], Richter [Ric66, Ric71], Sen [Sen71], Suzumura [Suz76], as well as many others - has serious difficulties dealing with social norms. The example we offered in the introduction of this article according to which a person must choose among fruits from a basket and contrary to her preferences does not choose the last apple from the basket is clearly an illustration in which a social norm of politeness is in operation.

Sen considers in [Sen97] various ways in which norms can be explicitly represented formally but ultimately does not manage to accommodate them systematically within the standard framework of the theory of rational choice. Nevertheless, recent work by Walter Bossert and Kotaro Suzumura [BS07] attempts to resolve the difficulties Sen has brought to the forefront. In this section we will present the main ideas behind Bossert and Suzumura's theory. We will also offer some criticisms. Nonetheless, we will later adopt some of the basic ideas upon which Bossert and Suzumura's framework is based.

In [BS07], Bossert and Suzumura seek to demonstrate that the rationalizability approach to the theory of choice remains robust in spite of the difficulties Sen raises concerning the influence of social norms. Introducing a notion of rationalizability they call norm-conditional rationalizability, Bossert and Suzumura extend the standard framework

\footnotetext{
${ }^{16}$ Interestingly, it seems that neither Rott nor AGM noticed that condition $\gamma \mathrm{R}_{\infty}$ is necessary and sufficient for rationalizability over general domains (i.e., domains for which no restrictions are imposed, such as closure under finite unions or compactness). Even more interesting is that to our knowledge, condition $\gamma \mathrm{R}_{\infty}$ has not appeared anywhere in the literature on choice functions. In particular, it appears that no one has explicitly pointed to a connection between condition $\gamma \mathrm{R}_{\infty}$ and rationalizability.

${ }^{17}$ Postulates $(\doteq 7)$ and $(\doteq 8 r)$ are supplementary postulates of belief contraction (see [AGM85] and [Rot93]).

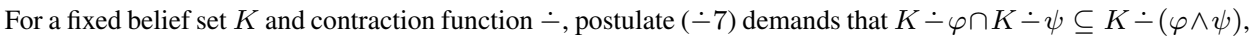
while postulate $(\doteq 8 r)$ requires that $K \dot{-}(\varphi \wedge \psi) \subseteq \mathrm{Cn}(K \dot{\doteq} \cup \cup K \dot{-} \psi)$.
} 
to cover some areas where it cannot be straightforwardly applied. We are sympathetic with this general idea.

In essence, Bossert and Suzumura develop an idea Sen briefly explores in [Sen97] to incorporate into standard rationalizability theory the influence of social norms in choice. According to Sen, some options from a menu of alternatives are excluded from permissible conduct through what he calls self-imposed choice constraints [Sen97, p. 769]. A person faced with a menu $S$ may first exclude some alternatives from $S$ by taking a permissible subset $K(S)$, which represents the person's self-imposed choice constraints, thereupon taking those alternatives from $K(S)$ which are 'best' or 'no worse' than the other alternatives from $K(S)$. Thus, Sen's self-styled permissibility function $K$ is such that for each menu $S, K(S)$ identifies a permissible subset of $S$. Bossert and Suzumura attempt to develop a formal framework to "bridge the idea of norm-induced constraints and the theory of rationalizability" [BS07, p. 10], a bridge for which Sen only originated its concept.

Let us for a moment return to the example from the introduction of this article to acquire an understanding of the rudiments of Bossert and Suzumura's approach. In this example, a person is faced with a choice between having the last remaining apple in the fruit basket $(y)$ and having nothing instead $(x)$. Faced with the decision problem represented by $\{x, y\}$, she decides to behave decently and picks nothing $(x)$. Yet if instead she had been confronted with the choice among having nothing $(x)$, having one nice apple $(y)$ and having another nice one $(z)$, she could reasonably enough choose an apple $(y)$, without violating any rule of good behavior. Thus, faced with the decision problem represented by $\{x, y, z\}$, she could reasonably choose $y$. Now if $\gamma$ is a selection function that characterizes these choices, we have that $\gamma(\{x, y\})=\{x\}$ while $\gamma(\{x, y, z\})=\{y\}$, which is clearly in violation of condition $\alpha$, thereby precluding rationalizability (see Proposition 1.4).

Bossert and Suzumura's framework explicitly represents the influence of norms in choice. Thus, in the foregoing example, the social norm enjoining that one should not choose the last available apple is represented simply by specifying that the choice of $y$ from $\{x, y\}$ is prohibited, whereas the choice of $y$ (or $z$ ) from $\{x, y, z\}$ is permissible. The norm so represented thereby takes into account the consequences of its application. Formally, a social norm is represented as a collection $\mathcal{N}$ of pairs of the form $(S, x)$, where $S$ is a menu and $x \in S$ is prohibited from being chosen from the set $S$.

Before we continue we should lay down the central components of Bossert and Suzmura's framework. We will do this using our notation and terminology as necessary. According to Bossert and Suzumura, a choice space $(X, \mathscr{S})$ must be such that $\emptyset \notin \mathscr{S}$, and a choice function is a regular selection function $\gamma: \mathscr{S} \rightarrow \mathscr{P}(X)$ such that $\gamma(S) \subseteq S \backslash\{x \in$ $S:(S, x) \in \mathcal{N}\}$ for all $S \in \mathscr{S}$. To ensure that the regularity requirement does not conflict with the constraints imposed by the norm $\mathcal{N}$, Bossert and Suzumura stipulate that $\mathcal{N}$ is such that for all $S \in \mathscr{S},\{S\} \times S \nsubseteq \mathcal{N}$, i.e., there exists $x \in S$ such that $(S, x) \notin \mathcal{N}$. Let us call a selection function on a choice space in the sense of Bossert and Suzumura a normative choice function.

We may now represent the foregoing example as follows. For $X:=\{x, y, z\}$ and $\mathscr{S}:=\{\{x, y, z\},\{x, y\}\}$, the operative social norm may be expressed by the set $\mathcal{N}:=$ $\{(\{x, y\}, y)\}$. Accordingly, since it is required that $\gamma(\{x, y\}) \subseteq\{x, y\} \backslash\{v \in\{x, y\}$ : $(\{x, y\}, v) \in \mathcal{N}\}$, the social norm demands that $y \notin \gamma(\{x, y\})$.

As indicated above, the primary goal in [BS07] is to develop a new concept of rationalizability called norm-conditional rationalizability in a effort to show that the standard rationalizability approach can accommodate the existence of social norms. Roughly, for a normative choice function $\gamma$ on a choice space $(X, \mathscr{S})$, norm-conditional rationalizability 
requires the existence of a preference relation such that for each menu $S \in \mathscr{S}, \gamma(S)$ consists of those alternatives which are at least as good as all alternatives from $S$, except for those alternatives prohibited by the social norm $\mathcal{N}$.

To make this precise, we need some notation and terminology, but the main idea is rather simple and direct. Let $\mathcal{N}$ be a social norm, let $(X, \mathscr{S})$ be a normative choice space, and let $S \in \mathscr{S}$. Bossert and Suzumura define an $\mathcal{N}$-admissible set for $(\mathcal{N}, S), A^{\mathcal{N}}(S)$, by setting

$$
A^{\mathcal{N}}(S):=\{x \in S:(S, x) \notin \mathcal{N}\} .
$$

Observe that according to Bossert and Suzumura's framework, for each $S \in \mathscr{S}, A^{\mathcal{N}}(S) \neq$ $\emptyset$ and $A^{\mathcal{N}}(S) \subseteq S$. Also observe that $A^{\mathcal{N}}(S)$ is a permissibility function in the sense of Sen.

Bossert and Suzumura then define norm-conditional rationalizability as follows (again adopting our notation and terminology as needed). A normative choice function $\gamma$ on a choice space $(X, \mathscr{S})$ is $\mathcal{N}$-rationalizable if there exists a binary relation $R^{\mathcal{N}}$ on $X$ such that for all $S \in \mathscr{S}$,

$$
\gamma(S):=\left\{x \in A^{\mathcal{N}}(S): x R^{\mathcal{N}} y \text { for all } y \in A^{\mathcal{N}}(S)\right\} .
$$

Thus the central idea is simple: for each menu $S \in \mathscr{S}, \gamma(S)$ selects the best alternatives from the $\mathcal{N}$-admissible set $A^{\mathcal{N}}(S)$.

In order to facilitate their analysis of norm-conditional rationalizability, Bossert and Suzumura utilize a generalization of the notion of so-called Samuelson preferences. Given a social norm $\mathcal{N}$ and a normative choice function $\gamma$ on a choice space $(X, \mathscr{S})$, Bossert and Suzumura define a binary relation on $X, R_{\gamma}$, called direct revealed preference, by setting

$$
R_{\gamma}:=\bigcup_{S \in \mathscr{S}}\left(\gamma(S) \times A^{\mathcal{N}}(S)\right) .
$$

Bossert and Suzumura then propose a generalization of a coherence constraint due to Richter [Ric71, p. 33]:

$\left(\mathcal{N}\right.$ drc) $\quad$ For all $S \in \mathscr{S}$ and $x \in A^{\mathcal{N}}(S)$, $(\mathcal{N}$-conditional direct-revelation coherence $)$ if for every $y \in A^{\mathcal{N}}(S) x R_{\gamma} y$, then $x \in \gamma(S)$.

In one of the central results of their article, Bossert and Suzumura prove that condition $\mathcal{N}$ drc is indeed necessary and sufficient for $\mathcal{N}$-rationalizability. The result is direct, and it is rather clear that this property is mathematically required in order to establish the result that Bossert and Suzumura desire. There are, nevertheless, some aspects of this result that we find unsatisfactory.

First, the condition in question, as many coherence constraints of this type used in the theory of rational choice (such as Suzumura's so-called Generalized Condorcet property [Suz83, p. 32]) establishes a constraint on a selection function $\gamma$ only indirectly by way of a constraint on $R_{\gamma}$. This type of condition not only incorporates a non-primitive notion but also is difficult to use in order to obtain mappings between selection functions and belief revision functions. Second - a somewhat related point --the condition proposed by Bossert and Suzumura offers little insight into the notion of $\mathcal{N}$-rationalizability in terms of the behavior of selection functions. Thus, we find it better to have a 'pure' constraint on $\gamma$ in the spirt of coherence constraints such as conditions $\alpha$ and $\gamma$.

Nonetheless, we find that Bossert and Suzumura have made a step in the right direction. First, they have developed a modified approach to rationalizability which presupposes no constraints on the domains of choice functions (except that domains cannot include the empty set). Second, because they have presupposed no restrictions on how norms come about, Bossert and Suzumura's approach is very general. Indeed, Bossert and Suzumura's 
theory of norm-conditional rationalizability is an extension of the classical theory of rationalizability, which is included as a special case.

In the following section, we will offer a refinement of Bossert and Suzumura's theory of norm-conditional rationalizability. We will also offer a coherence constraint that is 'pure.' Ultimately, we will see how to apply our theory to belief change in an effort to accommodate social norms in belief change.

2.2. Norm-Conditional Choice Models. In this section, we introduce what we call normconditional choice models. These models, inspired by both the ideas of Sen and the work of Bossert and Suzumura we discussed in the previous section, are intended to accommodate the role social norms play in choice. We reformulate Bossert and Suzumura's framework to improve upon the formal foundations upon which their theory of norm-condtional rationalizability is built. Among other things, our reformulation squares better with Sen's original conception of what he calls permissibility functions. In particular, we take permissibility functions as primitive. We offer several coherence constraints analogous to those discussed in Section 1.2. We also offer a 'pure' coherence constraint, which in the context of our framework, is both necessary and sufficient for norm-conditional rationalizability.

The reader will notice that we have borrowed several central concepts from Bossert and Suzumura's framework. Nonetheless, to avoid confusion, we have adopted a somewhat different notation and terminology, couching these concepts within our framework of norm-conditional choice models.

Definition 2.1. Let $\gamma$ and $\pi$ be selection functions on a choice space $(X, \mathscr{S})$. We call the pair $(\gamma, \pi)$ a norm-conditional choice model on $(X, \mathscr{S})$ if for every $S \in \mathscr{S}, \gamma(S) \subseteq \pi(S)$. We call $\gamma$ a $\pi$-conditional choice function, and we say that $\pi$ is a permissiblity function for $\gamma$.

Thus, in contrast with Bossert and Suzumura's framework, we take permissibility functions as primitive. We also do not prohibit $\emptyset \in \mathscr{S}, \gamma(S)=\emptyset$, or $\pi(S)=\emptyset$. We now directly borrow Bossert and Suzumura's notion of norm-conditional rationalizability.

Definition 2.2. Let $(\gamma, \pi)$ be norm-conditional choice model on $(X, \mathscr{S})$. We say that a binary relation $R$ on $X \pi$-rationalizes $\gamma$ if for every $S \in \mathscr{S}$,

$$
\gamma(S)=\{x \in \pi(S): x R y \text { for all } y \in \pi(S)\} .
$$

We also say that $\gamma$ is $\pi$-rationalizable if there is a binary relation $R$ on $X$ that $\pi$-rationalizes $\gamma$.

As with the standard theory of rationalizability, we can articulate notions of optimization and maximization for norm-conditional choice models.

Definition 2.3. Let $(\gamma, \pi)$ be a norm-conditional choice model on $(X, \mathscr{S})$.

(i) We say that $\gamma$ is $G \pi$-rational (or $G \pi$-rationalizable) if there is a reflexive binary relation $\geq$ on $X$ that $\pi$-rationalizes $\gamma$.

(ii) We say that $\gamma$ is $M \pi$-rational (or $M \pi$-rationalizable) if there is an asymmetric binary relation $>$ on $X$ such that $((X \times X) \backslash>)^{-1} \pi$-rationalizes $\gamma$.

The following coherence constraints impose conditions upon the interaction of the components of a norm-conditional choice model.

$\left(\gamma[\pi]_{>\emptyset}\right) \quad$ For each $S \in \mathscr{S}$, if $\pi(S) \neq \emptyset$, then $\gamma(S) \neq \emptyset$.

$\left(\gamma[\pi]_{1>\emptyset}\right) \quad$ For each $S \in \mathscr{S}$, if $|\pi(S)|=1$, then $\gamma(S) \neq \emptyset$.

$\left(\gamma[\pi] \mathrm{R}_{\infty}\right) \quad$ For every nonempty $I \subseteq \mathscr{S}$ and $S \in \mathscr{S}$, if $\pi(S) \subseteq \bigcup_{T \in I} \pi(T)$, then $\pi(S) \cap\left(\bigcap_{T \in I} \gamma(T)\right) \subseteq \gamma(S)$.
( $\pi$-Conditional Regularity) (Singleton $\pi$-Conditional Regularity) ( $\pi$-Conditional Coherence) 
As its name suggests, condition $\gamma[\pi]_{>\emptyset}$ is a hybrid analogue of condition $\gamma_{>\emptyset}$ from Section 1.2. A $\pi$-conditional choice function $\gamma$ satisfies condition $\gamma_{>\emptyset}$ just in case the normconditional choice model $(\gamma, \pi)$ satisfies condition $\gamma[\pi]_{>\emptyset}$ and the permissibility function $\pi$ for $\gamma$ satisfies condition $\gamma_{>\emptyset}$. As with condition $\gamma[\pi]_{>\emptyset}$, condition $\gamma[\pi]_{1>\emptyset}$ is a hybrid analogue of condition $\gamma_{1>\emptyset}$ from Section 1.2. Clearly condition $\gamma[\pi]_{>\emptyset}$ entails condition $\gamma[\pi]_{1>\emptyset}$. If $\pi$ is a permissibility function for $\gamma$ that satisfies condition $\gamma_{1>\emptyset}$, then $\gamma$ also satisfies condition $\gamma_{1>\emptyset}$ provided the norm-conditional choice model $(\gamma, \pi)$ satisfies condition $\gamma[\pi]_{1>\emptyset}$.

In the following, we say that a norm-conditional choice model $(\gamma, \pi)$ is $\pi$-conditional regular if $(\gamma, \pi)$ satisfies condition $\gamma[\pi]_{>\emptyset}$. We also call $(\gamma, \pi)$ regular if $\gamma$ is regular.

Observe that condition $\gamma[\pi] \mathbf{R}_{\infty}$ is an analogue of condition $\gamma \mathbf{R}_{\infty}$ from Section 1.2. Among other things, it entails hybrid versions of conditions $\alpha$ and $\gamma$.

$$
\begin{array}{lll}
(\gamma[\pi] \alpha) & \text { For every } S, T \in \mathscr{S}, \text { if } \pi(S) \subseteq \pi(T), \text { then } \pi(S) \cap \gamma(T) \subseteq \gamma(S) . & \text { (Norm-Conditional Contraction) } \\
(\gamma[\pi] \gamma) & \text { For every nonempty } I \subseteq \mathscr{S} \text { such that } \bigcup_{S \in I} S \in \mathscr{S}, & \text { (Norm-Conditional Expansion) } \\
& \text { if } \pi\left(\bigcup_{S \in I} S\right)=\bigcup_{S \in I} \pi(S), \text { then } \bigcap_{S \in I} \gamma(S) \subseteq \gamma\left(\bigcup_{S \in I} S\right) . &
\end{array}
$$

In this context, condition $\gamma[\pi] \alpha$ demands that any permissible alternative rejected for choice from a decision problem must remain rejected in any other menu for which the permissible options from the decision problem are permissible in the other menu.

Condition $\gamma[\pi] R_{\infty}$ has a somewhat complicated formulation. The reason for this is that we have not imposed any substantial constraints on the behavior of permissibility functions. Even if we assume that the underlying domain of a norm-conditional choice model satisfies constraints such as closure under finite unions and compactness, condition $\gamma[\pi] \mathbf{R}_{\infty}$ need not be reducible to simpler coherence constraints as condition $\gamma \mathbf{R}_{\infty}$ is reducible to conditions $\alpha^{*}$ and $\gamma^{*}$. Thus, a reduction of this sort would in part depend on conditions imposed on permissibility functions.

We have seen that condition $\gamma \mathbf{R}_{\infty}$ characterizes rationalizability. In particular, we have seen that this result holds without imposing any conditions on the domains of selection functions. Shortly we will witness a similar result with respect to norm-conditional models.

We consider one more coherence constraint that represents an interesting limit case.

$\left(\pi_{\iota}\right) \quad$ For each $S \in \mathscr{S}, S \subseteq \pi(S)$. (Norm in Absentia)

A permissibility function satisfying condition $\pi_{\iota}$ is devoid of any real influence on choice. So as one should expect, rationalizablity and norm-conditional rationalizability collapse.

Proposition 2.4. Let $(\gamma, \pi)$ be a norm-conditional choice model on $(X, \mathscr{S})$. Suppose $\gamma$ satisfies condition $\pi_{\iota}$. Then $\gamma$ is rational if and only if it is $\pi$-rational.

For our purposes we now borrow Bossert and Suzumura's notion of direct revealed preference.

Definition 2.5. Let $(\gamma, \pi)$ be a norm-conditional choice model on $(X, \mathscr{S})$. We define a binary relation $R_{\gamma}$ on $X$ by setting

$$
R_{\gamma}:=\bigcup_{S \in \mathscr{S}}(\gamma(S) \times \pi(S)) .
$$

We now present the central result of this section.

Theorem 2.6. Let $(\gamma, \pi)$ be a norm-conditional choice model on $(X, \mathscr{S})$. Then $\gamma$ is $\pi$ rational if and only if $(\gamma, \pi)$ satisfies condition $\gamma[\pi] \mathbf{R}_{\infty}$.

Proof. In the following, for a binary relation $R$ and $S \in \mathscr{S}$, let

$$
G(\pi(S), R):=\{x \in \pi(S): x R y \text { for all } y \in \pi(S)\} .
$$


$\Leftrightarrow$ Suppose $\gamma$ is $\pi$-rationalizable, and let $R \pi$-rationalize $\gamma$. We show that $(\gamma, \pi)$ satisfies $\gamma[\pi] \mathrm{R}_{\infty}$. Let $I \subseteq \mathscr{S}$ be such that $I \neq \emptyset$, let $S \in \mathscr{S}$, and suppose $\pi(S) \subseteq$ $\bigcup_{T \in I} \pi(T)$. Assume $x \in \pi(S) \cap\left(\bigcap_{T \in I} \gamma(T)\right)$. We show that $x \in G(\pi(S), R)$. We must only establish that for every $y \in \pi(S), x R y$. So let $y \in \pi(S)$. Then $y \in \bigcup_{T \in I} \pi(T)$, whereby $y \in \pi(T)$ for some $T \in I$. Observe that since $x \in$ $\bigcap_{T \in I} \gamma(T)$, we have that $x R z$ for each $T \in I$ and $z \in \pi(T)$, whence $x R y$. It follows that $x \in \gamma(S)$.

$(\Leftarrow)$ Suppose $(\gamma, \pi)$ satisfies condition $\gamma[\pi] \mathbf{R}_{\infty}$. We show that $R_{\gamma} \pi$-rationalizes $\gamma$. Let $S \in \mathscr{S}$.

$\gamma(S) \subseteq G\left(\pi(S), R_{\gamma}\right)$ : If $x \in \gamma(S)$, then $x \in \pi(S)$ and for every $y \in \pi(S)$, by definition $x R_{\gamma} y$; thus, $x \in G\left(\pi(S), R_{\gamma}\right)$.

$G\left(\pi(S), R_{\gamma}\right) \subseteq \gamma(S)$ : Let $x \in G\left(\pi(S), R_{\gamma}\right)$. Then $x \in \pi(S)$ and for every $y \in$ $\pi(S), x R_{\gamma} y$, so for every $y \in \pi(S)$, there is $T_{y} \in \mathscr{S}$ such that $x \in \gamma\left(T_{y}\right)$ and $y \in$ $\pi\left(T_{y}\right)$. Observe that since $\pi(S) \subseteq \bigcup_{y \in \pi(S)} \pi\left(T_{y}\right)$, by condition $\gamma[\pi] \mathbf{R}_{\infty}$ we have that $\pi(S) \cap\left(\bigcap_{y \in \pi(S)} \gamma\left(T_{y}\right)\right) \subseteq \gamma(S)$. Hence, since $x \in \pi(S) \cap\left(\bigcap_{y \in \pi(S)} \gamma\left(T_{y}\right)\right)$, it follows that $x \in \gamma(S)$, as desired.

In Section 1.2 we showed that condition $\gamma_{1>\emptyset}$ is necessary for G-rationality. We have a similar result for condition $\gamma[\pi]_{1>\emptyset}$.

Theorem 2.7. Let $(\gamma, \pi)$ be a norm-conditional choice model on $(X, \mathscr{S})$. Then $\gamma$ is $G \pi$ rational if and only if $(\gamma, \pi)$ satisfies condition $\gamma[\pi] \mathbf{R}_{\infty}$ and condition $\gamma[\pi]_{1>\emptyset}$.

Proof.

$\Leftrightarrow$ Suppose $\gamma$ is $G \pi$-rationalizable, and let $\geq$ be a reflexive binary relation on $X$ that $\pi$-rationalizes $\gamma$. By Theorem 2.6, $(\gamma, \pi)$ satisfies condition $\gamma[\pi] \mathbf{R}_{\infty}$. We show that $(\gamma, \pi)$ satisfies condition $\gamma[\pi]_{1>\emptyset}$. For reductio ad absurdum, assume there is $S \in \mathscr{S}$ such that $|\pi(S)|=1$, but $\gamma(S)=\emptyset$. Then there is $x \in S$ such that $\pi(S)=\{x\}$, but since $\geq \pi$-rationalizes $\gamma$, it follows that $x \nsupseteq x$, contradicting that $\geq$ is reflexive.

$(\Leftarrow)$ Suppose $(\gamma, \pi)$ satisfies condition $\gamma[\pi] \mathbf{R}_{\infty}$ and condition $\gamma[\pi]_{1>\emptyset}$. By the proof of Theorem 2.6, $R_{\gamma} \pi$-rationalizes $\gamma$. We show that there is a reflexive binary relation on $X$ that $\pi$-rationalizes $\gamma$. Let $\Delta$ be the diagonal of $X$ (i.e., $\Delta:=\{(x, x): x \in$ $X\}$ ). Define a binary relation $\geq$ on $X$ by setting

$$
\geq:=R_{\gamma} \cup \Delta
$$

Clearly $\geq$ is reflexive. We show that $\geq \pi$-rationalizes $\gamma$. Let $S \in \mathscr{S}$. In the following, let

$$
G(\pi(S), \geq):=\{x \in \pi(S): x \geq y \text { for all } y \in \pi(S)\} .
$$

$\gamma(S) \subseteq G(\pi(S), \geq)$ : Suppose $x \in \gamma(S)$. Then since $R_{\gamma} \pi$-rationalizes $\gamma$, we have that $x \in \pi(S)$ and for every $y \in \pi(S), x R_{\gamma} y$, whence for every $y \in \pi(S)$, $x \geq y$. Thus, $x \in G(\pi(S), \geq)$.

$G(\pi(S), \geq) \subseteq \gamma(S)$ : Suppose $x \in G(\pi(S), \geq)$. Then $x \in \pi(S)$ and for every $y \in \pi(S), x \geq y$, so for every $y \in \pi(S)$ for which $x \neq y, x R_{\gamma} y$. Now if $|\pi(S)|=1$, by condition $\gamma[\pi]_{1>\emptyset}$ we have that $\gamma(S) \neq \emptyset$, whereby since $\gamma$ is $\pi$-rational, it follows that $x \in \gamma(S)$, and we are done. So suppose $|\pi(S)|>1$. Then there is $y \in \pi(S)$ such that $x \neq y$ and $x \geq y$ and so $x R_{\gamma} y$, whereupon it follows that for some $T \in \mathscr{S}, x \in \gamma(T)$. Therefore, since $R_{\gamma} \pi$-rationalizes $\gamma$, 
we have that $x R_{\gamma} x$. Hence, for every $y \in \pi(S), x R_{\gamma} y$, so since $R_{\gamma} \pi$-rationalizes $\gamma$, we have that $x \in \gamma(S)$, as desired.

We thereby have the following corollary.

Corollary 2.8. Let $(\gamma, \pi)$ be a norm-conditional choice model on $(X, \mathscr{S})$. Suppose $\gamma$ satisfies condition $\gamma_{>\emptyset}$ or condition $\gamma[\pi]_{>\emptyset}$. Then $\gamma$ is $G \pi$-rational if and only if $(\gamma, \pi)$ satisfies condition $\gamma[\pi] \mathbf{R}_{\infty}$.

We have seen in this section that condition $\gamma[\pi] \mathbf{R}_{\infty}$ characterizes norm-conditional rationalizability. This result holds without having presupposed conditions on the domains underlying norm-conditional choice models or on the components of norm-conditional choice models. Our results are therefore comparable to those of Bossert and Suzumura. Yet we have also refined Bossert and Suzumura's framework, and in particular, we have offered, as promised, a coherence constraint that is 'pure' in the spirit of conditions such as $\alpha$ and $\gamma$.

This completes our proposal regarding social norms in the realm of rational choice. Now we turn to epistemology and the notion of belief revision, where we will soon see how the foregoing results can be fruitfully applied. In the next section it will be become apparent that conditions $\gamma \mathbf{R}_{\infty}, \gamma \mathbf{R}_{<\omega}$, and $\gamma \mathbf{R}_{1}$ have natural translations into postulates we label $\left(* \mathrm{R}_{\infty}\right),\left(* \mathrm{R}_{<\omega}\right)$, and $\left(* \mathrm{R}_{1}\right)$. Later we will see that just as these conditions have natural translations into postulates of belief revision, condition $\gamma[\pi] \mathbf{R}_{\infty}$ corresponds to a central postulate of what we will call norm-inclusive belief revision.

\section{PART III: Belief ReVision}

Belief change has been formalized in several frameworks. In this article, the general framework of belief change under discussion is based on the work of Alchourrón, Gärdenfors, and Makinson (AGM) [AGM85]. We will presume familiarity with the AGM framework, but here we will review some of the basic ideas. ${ }^{18}$

In the AGM framework, an agent's belief state is represented by a logically closed set of sentences $K$, called a belief set. The sentences of $K$ are intended to represent the beliefs held by the agent. Belief change then comes in three flavors: expansion, revision, and contraction.

In expansion, a sentence $\varphi$ is added to a belief set $K$ to obtain an expanded belief set $K+\varphi$. This expanded belief set $K+\varphi$ might be logically inconsistent. In revision, by contrast, a sentence $\varphi$ is added to a belief set $K$ to obtain a revised belief set $K * \varphi$ in a way that preserves logical consistency. To ensure that $K * \varphi$ is consistent, some sentences from $K$ might be removed. In contraction, a sentence $\varphi$ is removed from $K$ to obtain a contracted belief set $K \dot{-} \varphi$ that does not include $\varphi$. In this article we will be primarily concerned with belief revision.

3.1. Postulates for Belief Revision. For a fixed belief set $K$, the following are the six basic postulates of belief revision ([AGM85, p. 513],[Han99, p. 212]):

\footnotetext{
${ }^{18} \mathrm{~A}$ comprehensive introduction to theories of belief change is [Han99]. A brief introduction to belief change may be found in [Gär92].
} 
(*1) $K * \varphi$ is a belief set.

$(* 2) \quad \varphi \in K * \varphi$.

(*3) $K * \varphi \subseteq \operatorname{Cn}(K \cup\{\varphi\})$.

(*4) If $\neg \varphi \notin K$, then $\operatorname{Cn}(K \cup\{\varphi\}) \subseteq K * \varphi$.

(*5) If $\operatorname{Cn}(\{\varphi\}) \neq \operatorname{For}(\mathcal{L})$, then $K * \varphi \neq \operatorname{For}(\mathcal{L})$.

(*6) If $\operatorname{Cn}(\{\varphi\})=\operatorname{Cn}(\{\psi\})$, then $K * \varphi=K * \psi$.
(Closure)

(Success)

(Inclusion)

(Vacuity)

(Consistency)

(Extensionality)

Let us henceforth call a function $*_{K}: \operatorname{For}(\mathcal{L}) \rightarrow \mathbb{K}$ a revision function over $K$ if it satisfies postulates $(* 1),(* 2)$, and $(* 6)$. Of course, we write $K * \varphi$ instead of $*_{K} \varphi$.

The six basic postulates are elementary requirements of belief revision and taken by themselves are much too permissive. Invariably, several postulates are added to the basic postulates to rein in this permissiveness and to add structure to belief change. Such postulates are called supplementary postulates. Among the various postulates added to the mix, the following postulate-or some equivalent or stronger version of it — never fails to be set forth [Gär79, p. 393]:

$(* 7 g) K * \varphi \cap K * \psi \subseteq K *(\varphi \vee \psi)$.

In [Han99, p. 217], postulate $(* 7 g)$ is called Disjunctive Overlap. ${ }^{19}$ It encodes the intuitive idea that if an agent believes $\delta$ whether it revises its beliefs $K$ by $\varphi$ or by $\psi$, then the agent ought to believe $\delta$ if the agent revises its beliefs $K$ by $\varphi \vee \psi$. In [Gär88, pp. 211-212], Peter Gärdenfors shows that in the presence of postulates $(* 1)-(* 6)$, postulate $(* 7 g)$ is equivalent to the following postulate:

$(* 7) K *(\varphi \wedge \psi) \subseteq \operatorname{Cn}((K * \varphi) \cup\{\psi\})$.

In fact, an examination of the proof in [Gär88] reveals that this equivalence holds even in the presence of only postulates $(* 1),(* 2)$, and $(* 6)$, i.e., if $*$ is revision function over $K$.

Often another postulate — or some postulate at least as strong as it—is added to the mix:

$(* 8 r) K *(\varphi \vee \psi) \subseteq \operatorname{Cn}(K * \varphi \cup K * \psi)$.

This postulate is called Disjunction in [GR95, p. 54]. ${ }^{20}$

As with conditions $\alpha^{*}$ and $\gamma^{*}$, we can combine postulates $(* 7 g)$ and $(* 8 r)$ into one postulate:

$(* \mathrm{R}) K * \varphi \cap K * \psi \subseteq K *(\varphi \vee \psi) \subseteq \mathrm{Cn}(K * \varphi \cup K * \psi)$.

If $*$ is a revision function over $K$, postulate $(* \mathrm{R})$ is equivalent to each of the following postulates:

$\left(* \mathrm{R}_{<\omega}\right)$ For every $n<\omega$,

if $\bigcap_{i<n} \operatorname{Cn}\left(\left\{\psi_{i}\right\}\right) \subseteq \operatorname{Cn}(\{\varphi\})$, then $K * \varphi \subseteq \operatorname{Cn}\left(\left(\bigcup_{i<n} K * \psi_{i}\right) \cup\{\varphi\}\right)$.

$\left(* \mathrm{R}_{1}\right)$ If $\psi_{0} \vee \psi_{1} \in \mathrm{Cn}(\{\varphi\})$, then $K * \varphi \subseteq \operatorname{Cn}\left(K * \psi_{0} \cup K * \psi_{1} \cup\{\varphi\}\right)$.

Furthermore, if $\mathcal{L}$ is finite and $*$ is a revision function over $K$, postulate $(* \mathrm{R})$ is equivalent to the following postulate:

$\left(* \mathrm{R}_{\infty}\right)$ For every nonempty $I \subseteq \operatorname{For}(\mathcal{L})$, if $\bigcap_{\psi \in I} \operatorname{Cn}(\{\psi\}) \subseteq \operatorname{Cn}(\{\varphi\})$, then $K * \varphi \subseteq \operatorname{Cn}\left(\left(\bigcup_{\psi \in I} K * \psi\right) \cup\{\varphi\}\right)$.

We record these observations in a proposition, leaving the proof to the reader:

Proposition 3.1. Let $*$ be a revision function over $K$. Then $(* R),\left(* R_{<\omega}\right)$, and $\left(* R_{1}\right)$ are pairwise equivalent. If in addition $\mathcal{L}$ is finite, then $\left(* \mathrm{R}_{\infty}\right)$ is pairwise equivalent to each of the aforementioned postulates.

\footnotetext{
${ }^{19}$ The ' $\mathrm{g}$ ' in $(* 7 g)$ is for 'Gärdenfors' [Rot01, p. 110].

${ }^{20}$ Rott labels this postulate $(* 8 v w d)$ in [Rot 01, p. 110].
} 
3.2. Selection Functions in Belief Revision. The major innovation in [AGM85] is the employment of selection functions to define operators of belief change. In [AGM85], selection functions take remainder sets as arguments. ${ }^{21}$ In this article we utilize selection functions which take propositions expressed by formulae as arguments, i.e., selection functions on the choice space $\left(\mathcal{W}_{\mathcal{L}}, \mathcal{E}_{\mathcal{L}}\right)$ (see Section 1.1; see also [Gro88]). Such selection functions are called semantic selection functions. Rott has shown in [Rot01] that this approach is a fruitful generalization of the AGM approach.

Optimization, called strong maximization in [GR95, p. 65], is put to use in the classical AGM theory of belief change [AGM85]. There a selection function chooses the remainders of a remainder set that are 'best' in the sense that they are most worth retaining according to some non-strict ordering (the so-called 'marking-off' relation in [Han99, p. 82]). ${ }^{22}$

It is also possible to apply maximization to study belief change. This notion, called weak maximization in [GR95, p. 65], is explored at length by the first author in [Arl06], and Rott advocates using this notion in [Rot93, p. 1430] and [Rot01, p. 156]. Indeed, there are good reasons to believe that this formalization is superior to the aforementioned formalization. Here we take a neutral position with respect to this issue, and most of our discussion about belief change will be framed with respect to optimization.

We point to a simple formal connection between rational choice on the one hand and belief change and non-monotonic reasoning on the other. In rational choice, G-rational and M-rational selection functions are often called rationalizable. However, in the study of belief change and non-monotonic reasoning, G-rational (i.e., strongly rationalizable) and M-rational (i.e., weakly rationalizable) selection functions are often called relational. Thus, formally speaking, rationalizablity in rational choice is equivalent to relationality in belief change and non-monotonic reasoning.

3.3. Rott's Correspondence Results. In this section we will review Rott's correspondence results linking conditions of belief revision and coherence postulates in the theory of rational choice. We will present his results in a way that brings out their bearing upon rationalizabillty in belief change. We begin with several definitions (at this point the reader may wish to review Section 1.1).

Definition 3.2. A semantic selection function is a selection function on choice space $\left(\mathcal{W}_{\mathcal{L}}, \mathcal{E}_{\mathcal{L}}\right)$.

Recall that $\mathcal{W}_{\mathcal{L}}$ denotes the collection of all maximal consistent sets of $\mathcal{L}$ with respect to $\mathrm{Cn}$, while $\mathcal{E}_{\mathcal{L}}$ denotes the set of all elementary subsets of $\mathcal{W}_{\mathcal{L}}$.

Definition 3.3. Let $\gamma$ be a semantic selection function.

(i) We define a semantic selection function $\bar{\gamma}$ by setting for all $S \in \mathcal{E}_{\mathcal{L}}$,

$$
\bar{\gamma}(S):= \begin{cases}\llbracket \widehat{\llbracket(S)} \rrbracket & \text { if } \gamma(S) \neq \emptyset \\ \emptyset & \text { otherwise. }\end{cases}
$$

\footnotetext{
${ }^{21}$ For a belief set $K$ and a sentence $\varphi$, a remainder set $K \perp \varphi$ is the set of maximal consistent subsets of $K$ that do not imply $\varphi$. Members of $K \perp \varphi$ are called remainders. Thus, in the AGM framework, a belief set $K$ is fixed, and for every sentence $\varphi$ such that $\varphi \notin \operatorname{Cn}(\emptyset), \gamma(K \perp \varphi)$ selects a set of remainders of $K \perp \varphi$. The situation in which $\varphi \in \mathrm{Cn}(\emptyset)$ can be handled as a limiting case at the level of the selection function [AGM85] or at the level of the revision operator [Rot93].

${ }^{22}$ In [AGM85, pp. 517-518], a relation $\geq$ is defined over remainder sets for a fixed belief set $K$, and $\mathrm{Eq}_{\geq}$is called the marking off identity:$$
\gamma(K \perp \varphi)=\left\{B \in K \perp \varphi: B \geq B^{\prime} \text { for all } B^{\prime} \in K \perp \varphi\right\} .
$$ 
We call $\bar{\gamma}$ the completion of $\gamma$.

(ii) We say that $\gamma$ is complete if $\gamma=\bar{\gamma}$.

Observe that for every $S \in \mathcal{E}_{\mathcal{L}}, \bar{\gamma}(S) \subseteq S$, so $\bar{\gamma}$ is a selection function. Also observe that for all $S \in \mathcal{E}_{\mathcal{L}}, \gamma(S) \subseteq \bar{\gamma}(S)$. Finally, observe that if $\mathcal{L}$ is finite, then every semantic selection function is complete.

We now define choice-based revision functions.

Definition 3.4. Let $K$ be a belief set, and let $\gamma$ be a semantic selection function. The semantic choice-based revision function $*$ over $K$ generated by $\gamma$ is defined by setting for every $\varphi \in \operatorname{For}(\mathcal{L})$,

$$
K * \varphi:= \begin{cases}\widehat{\gamma(\llbracket \varphi \rrbracket)} & \text { if } \gamma(\llbracket \varphi \rrbracket) \neq \emptyset \\ \operatorname{For}(\mathcal{L}) & \text { otherwise. }\end{cases}
$$

We say that $\gamma$ generates $*$ or that $*$ is generated by $\gamma$.

To bring the ideas concerning rationalizablity to the foreground, we offer the following definition.

Definition 3.5. Let $K$ be a belief set. We call a function $*$ a (regular, rational, G-rational, complete) choice-based revision function over $K$ if there is a (regular, rational, G-rational, complete) semantic selection function $\gamma$ that generates $*$.

Observe that every semantic choice-based revision function over a belief set $K$ satisfies postulates $(* 1),(* 2)$, and $(* 6)$ and so is indeed a revision function over $K$. It is an easy matter to check that the converse holds as well: If $*$ is a revision function over a belief set $K$, then $*$ is a semantic choice-based revision function over $K$.

Also observe that $*$ is a semantic choice-based function over $K$ generated by $\gamma$ if and only if for every sentence $\psi$ of $\mathcal{L}$,

$$
\psi \in K * \varphi \text { if and only if } \gamma(\llbracket \varphi \rrbracket) \subseteq \llbracket \psi \rrbracket .
$$

Intuitively, an agent believes a sentence $\psi$ in the revision of $K$ by $\varphi$ just in case $\psi$ is true in all the most 'plausible' worlds in which $\varphi$ is true. Of course, the role of a semantic selection function-or any selection function — can be interpreted in various ways in different contexts.

In [Rot01], Rott discusses a handful of coherence constraints for selection functions, some of which are well-known and others of which he debuts. We present two conditions of the latter sort without offering motivation (see [Rot01, pp. 147-149] for such motivation):

$\left(\mathrm{F} 1_{B}\right) \quad$ For every $S \in \mathscr{S}$, if $S \cap B \neq \emptyset$, then $\gamma(S) \subseteq B$. (Faith 1 respect to $B$ )

$\left(\mathrm{F} 2_{B}\right) \quad$ For every $S \in \mathscr{S}, S \cap B \subseteq \gamma(S)$. (Faith 2 respect to $B$ )

Let us now see how some of the coherence constraints-especially condition $\alpha$-are intimately connected with the presumption that selection functions are rationalizable in the study of belief change. Here we turn to Rott's recent correspondence results. Among other things, Rott's recent results establish a connection between condition $\alpha$ and postulate $(* 7)$ of belief revision. ${ }^{23}$ Presented in a form suitable for this article, the following theorem provides one part of this connection [Rot01, p. 197].

\footnotetext{
${ }^{23}$ Rott's results in [Rot01] show much more. For example, Rott shows that condition $\alpha$ corresponds not only to posutlate $(* 7)$, but also to postulate $(\dot{-} 7)$ of belief contraction (which requires that $K \dot{-} \varphi \cap K \dot{-} \psi \subseteq$ $K \dot{-}(\varphi \wedge \psi))$ [Rot01, pp. 193-196] and to rule (Or) of non-monotonic reasoning (which demands observance of the following: From $\varphi \sim \chi$ and $\psi \sim \chi$, infer $\varphi \vee \psi \sim \chi$ ) [Rot01, pp. 201-204].
} 
Theorem 3.6 ([Rot01]). Let $K$ be a belief set. For every semantic selection function $\gamma$ which satisfies

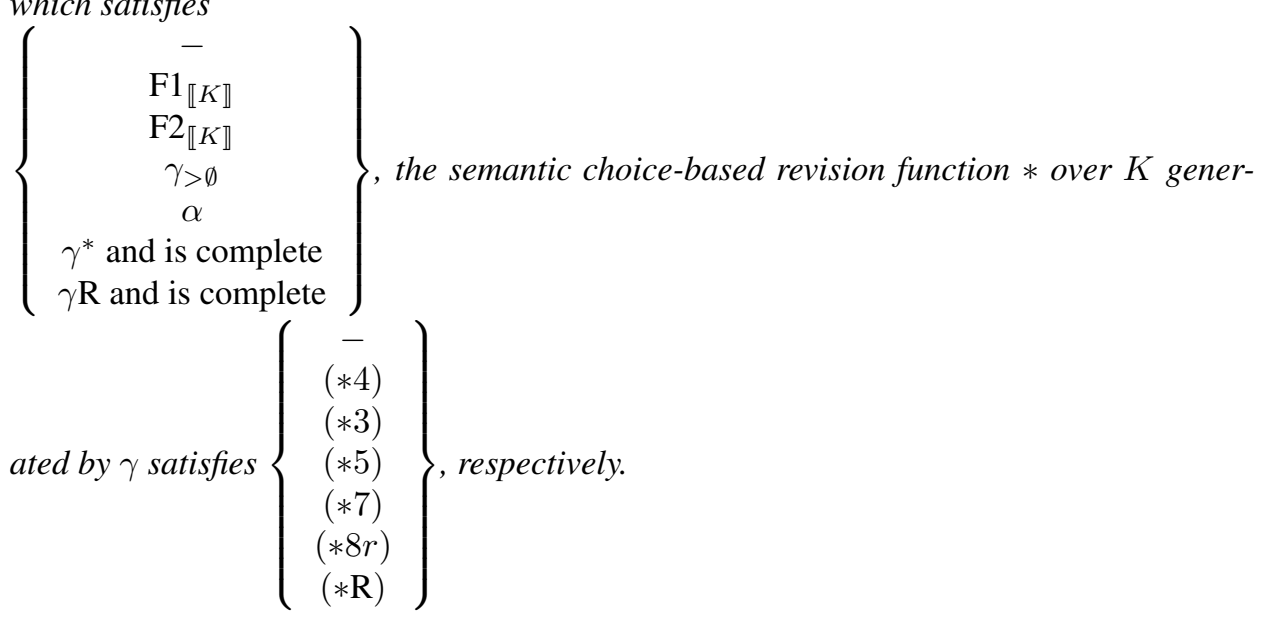

Theorem 3.6 is a 'soundness' result. (The reader should observe the modular character of Theorem 3.6 as well as Theorem 3.7 below. Theorem 3.6, for example, says that for every belief set $K$ and semantic selection function $\gamma$, if $\gamma$ satisfies condition $\mathrm{F} 1_{\llbracket K \rrbracket}$, then the semantic choice-based revision function $*$ over $K$ generated by $\gamma$ satisfies postulate $(* 4)$; Theorem 3.6 also says that for every belief set $K$ and semantic selection function $\gamma$, if $\gamma$ is complete and satisfies condition $\alpha$, then the semantic choice-based revision function $*$ over $K$ generated by $\gamma$ satisfies postulate $(* 7)$.) Rott also establishes a number of 'completeness' results. Also presented in a form suitable for this article, the following completeness result is the other part of the connection between coherence constraints and rationality postulates of belief revision [Rot01, p. 198].

Theorem 3.7 ([Rot01]). Every revision function $*$ over a belief set $K$ which satisfies
$\left.\begin{array}{c}- \\ (* 3) \\ (* 4) \\ (* 5) \\ (* 7) \\ (* 8) \\ (* \mathrm{R})\end{array}\right\}$ can be represented as the semantic choice-based revision function over $K$
generated by a semantic selection function $\gamma$ which satisfies $\left\{\begin{array}{c}- \\ \mathrm{F} 2_{\llbracket K \rrbracket} \\ \mathrm{F} 1_{\llbracket K \rrbracket} \\ \gamma>\emptyset \\ \alpha \\ \gamma^{*} \\ \gamma \mathrm{R}\end{array}\right\}$, respectively.

Observe that the preceding theorems do not presuppose any basic postulates other than $(* 1),(* 2)$, and $(* 6)$. Since $\left(\mathcal{W}_{\mathcal{L}}, \mathcal{E}_{\mathcal{L}}\right)$ is closed under finite unions and relative complements and is compact, we can apply the results from the previous section to obtain the following corollaries which are of particular relevance for the purposes of this article. ${ }^{24}$

\footnotetext{
${ }^{24}$ Sen's Property $\beta^{+}$was mentioned in footnote 11 because it has been given special attention in connection to rationalizablity in belief change and non-monotonic reasoning (e.g., in [Rot93], [Rot01], and [Ar106]). It is
} 
Corollary 3.8. Let $K$ be a belief set.

(i) Every rational choice-based revision function $*$ over $K$ is a revision function satisfying $(* 7)$, and every rational complete choice-based revision function $*$ over $K$ satisfies $(* 7)$ and $(* 8 r)$.

(ii) Every revision function $*$ over $K$ satisfying $(* 7)$ and $(* 8 r)$ is a rational (complete) choice-based revision function over $K$.

Corollary 3.9. Let $K$ be a belief set.

(i) Every regular G-rational choice-based revision function $*$ over $K$ is a revision function satisfying $(* 5)$ and $(* 7)$, and every regular $G$-rational complete choicebased revision function $*$ over $K$ satisfies $(* 5),(* 7)$, and $(* 8 r)$.

(ii) Every revision function $*$ over $K$ satisfying $(* 5),(* 7)$, and $(* 8 r)$ is a regular (complete) G-rational choice-based revision function over $K$.

We remark that if $\mathcal{L}$ is infinite, then for no $w \in \mathcal{W}_{\mathcal{L}}$ is it the case that $\{w\} \in \mathcal{E}_{\mathcal{L}}$. Thus, every selection function on $\left(\mathcal{W}_{\mathcal{L}}, \mathcal{E}_{\mathcal{L}}\right)$ satisfies $\gamma_{1>\emptyset}$. It follows that if $\mathcal{L}$ is infinite, then one can drop the requirement of regularity in Corollary 3.9. Yet if $\mathcal{L}$ is finite, one must impose regularity to guarantee G-rationality.

Corollary 3.10. Let $\mathcal{L}$ be finite, and let $K$ be a belief set.

(i) A function $*$ is a rational choice-based revision function over $K$ if and only if it is a revision function satisfying $(* 7)$ and $(* 8 r)$.

(ii) A function $*$ is a regular $G$-rational choice-based revision function over $K$ if and only if it is a revision function satisfying $(* 5),(* 7)$, and $(* 8 r)$.

With respect to rationalizability, the foregoing results are quite general. As indicated above, the only basic postulates presumed to hold in these results are $(* 1),(* 2)$, and $(* 6)$ (rationality postulates and coherence constraints can be added and subtracted modularly). According to the results, if there is a rational selection function $\gamma$ that generates a belief revision function $*$, then $*$ must satisfy postulate $(* 7)$. In the next section, we will offer plausible examples in which postulate $(* 7)$ is violated. And this should raise eyebrows: For such violations imply that no rational selection function exists that models the agent's belief change, and a fortiori, no G-rational or M-rational selection function exists that models the belief change. Thus, the universal presumption in the study of belief change and non-monotonic reasoning - that selection functions are rationalizable-must be called into question.

But simply abandoning this presumption is much too quick and much too damaging. The utilization of rationalizable selection functions in the study of belief change and nonmonotonic reasoning has proved to be quite useful and indeed indispensable, so it would be valuable to see what can be salvaged from the theoretical wreck.

We claim that the phenomenon of menu dependence-in epistemic form-is to blame for these violations of $(* 7)$. The counterexamples we offer in this article illustrate the role of menu dependence in the context of belief change and non-monotonic reasoning. We will indicate what formal measures may be taken to anticipate menu dependence, which nonetheless admit a restricted form of optimization and maximization.

known that condition $\beta^{+}$corresponds to belief revision's rationality postulate $(* 8)$, which requires that $K * \varphi \subseteq$ $K *(\varphi \wedge \psi)$ whenever $\neg \psi \notin K * \varphi$ (see [Rot01, p. 198]). But since condition $\beta$ is more pervasive in the study of rational choice, one might ask the following question: What rationality postulate corresponds to condition $\beta$ ? The second author has shown elsewhere [Ped08] that condition $\beta$ corresponds to postulate $(* 8 \beta)$, which demands that if $\operatorname{Cn}(K * \varphi \cup K *(\varphi \wedge \psi)) \neq \operatorname{For}(\mathcal{L})$, then $K * \varphi \subseteq K *(\varphi \wedge \psi)$. 
3.4. Counterexamples. In this section we will offer counterexamples to postulate $(* 7)$. Actually, these counterexamples violate many postulates of belief revision, just as counterexamples à la Sen violate many coherence constraints of rational choice, including condition $\alpha$.

Each counterexample involves three hypothetical scenarios in which an agent accepts belief-contravening information. Each scenario describes a potential unfolding of events. The scenarios in the counterexamples are not consecutive stages of a single chain of events. Rather, each scenario describes one way things could turn out. Moreover, only one of these scenarios will be realized.

Following each example, we will indicate how postulate $(* 7)$ fails. The first example is essentially a reproduction of an example presented by Rott in [Rot04]. We will see that menu dependence plays an essential role in this example. Then we will introduce variants of Rott's example where menu dependence can be explained in terms of the intervention of social norms.

3.4.1. Example. A philosophy department has announced an open position in metaphysics. Tom, an interested bystander, happens to know a few of the applicants: Amanda Andrews, Bernice Becker, Carlos Cortez, and Don Doyle. Tom, just like everyone else, knows that Andrews is an outstanding specialist in metaphysics, whereas Becker, who is also a very good metaphysician, is not quite as excellent as Andrews. However, Becker has done some substantial work in logic. Cortez has a comparatively slim record in metaphysics, yet he is widely recognized as one of the most brilliant logicians of his generation. By contrast, Doyle is a star metaphysician, while Andrews has done close to no work in logic.

Now suppose Tom initially believes that neither Andrews, Becker, nor Cortez will be offered the position because he, like everyone else, believes that Doyle is the obvious candidate to be offered the position. Tom is well-aware that only one of the applicants will be offered the position. Let $a, b, c$, and $d$ stand for the following sentences:

$a$ : Andrews will be offered the position.

$b$ : Becker will be offered the position.

$c$ : Cortez will be offered the position.

$d$ : Doyle will be offered the position.

Tom is having lunch with the dean. The dean is a very competent, serious, and honest man. $\mathrm{He}$ is also the chairman of the selection committee.

Scenario 1. The dean informs Tom that either Andrews or Becker will be offered the position. That is, the dean informs Tom that $a \vee b$. Because Tom presumes that expertise in metaphysics is the decisive criterion for the selection committee's decision, Tom concludes that Andrews will be offered the position (and of course that all other applicants will not be offered the position).

Scenario 2. The dean confides to Tom that either Andrews, Becker, or Cortez will be offered the position, thereby supplying him with $a \vee b \vee c$. Because Cortez is a brilliant logician, Tom realizes that he cannot sustain his presumption that metaphysics is the decisive criterion for the selection committee's decision. From Tom's perspective, logic also appears to be regarded as a considerable asset by the selection committee. Nonetheless, because Cortez has such a slim record in metaphysics, Tom believes that Cortez will not be offered the position. But Tom sees that logic contributes to an applicant's chances of being offered a position. 
Tom thereby concludes that Becker will be offered the position (and so no other applicant will be offered the position).

Scenario 3. The dean tells Tom that Cortez will be offered the position, thereby supplying him with $c$. Tom is certainly surprised, yet he believes what the dean tells him.

Let us take stock of Tom's beliefs in these scenarios. Initially, Tom believes $d, \neg a, \neg b$, and $\neg c$. Thus, letting $K$ denote Tom's initial belief set, $d, \neg a, \neg b$ and $\neg c$ are in $K$. In Scenario 1, Tom's revises his belief set $K$ by $a \vee b$, and his revised belief set $K *(a \vee b)$ contains $a$ and $\neg b$, as well as $\neg c$ and $\neg d$. In Scenario 2, Tom revises his belief set $K$ by $a \vee b \vee c$. His revised belief set $K *(a \vee b \vee c)$ includes $b, \neg a, \neg c$, and $\neg d$. Finally, in Scenario 3, Tom revises his belief set $K$ by $c$, whereby his revised belief set $K * c$ contains $c, \neg a, \neg b$, and $\neg d$.

We are now in a position to see that Example 3.4.1 constitutes a violation of postulate $(* 7)$. First, observe that $\neg b \in K *(a \vee b) \cap K * c$ and $\neg b \notin K *(a \vee b \vee c)$. Hence,

$$
K *(a \vee b) \cap K * c \nsubseteq K *(a \vee b \vee c)
$$

So postulate $(* 7 g)$ and therefore postulate $(* 7)$ is violated.

In light of Theorem 3.6, we should be unsurprised to see that condition $\alpha^{*}$ is also violated. And it is. Let $\gamma$ be any semantic selection function that generates $*$. Then it must be the case that $\gamma(\llbracket a \vee b \vee c \rrbracket) \subseteq \llbracket \neg a \wedge b \wedge \neg c \wedge d \rrbracket, \gamma(\llbracket a \vee b \rrbracket) \subseteq \llbracket a \wedge \neg b \wedge \neg c \wedge d \rrbracket$, and $\gamma(\llbracket c \rrbracket) \subseteq \llbracket \neg a \wedge \neg b \wedge c \wedge \neg d \rrbracket$. It must further be the case that $\gamma(\llbracket a \vee b \vee c \rrbracket) \nsubseteq \llbracket \neg b \rrbracket$, whereby $\gamma(\llbracket a \vee b \vee c \rrbracket) \cap \llbracket b \rrbracket \neq \emptyset$. It follows that

Thus, condition $\alpha^{*}$ is violated..$^{25}$

$$
\gamma(\llbracket a \vee b \vee c \rrbracket) \nsubseteq \gamma(\llbracket a \vee b \rrbracket) \cup \gamma(\llbracket c \rrbracket) .
$$

The phenomenon of menu dependence seems to explain the choices made in this case. When Tom faces the menu represented by $a \vee b$, he does it under the presumption that metaphysics is the decisive criterion for the selection committee's decision. Therefore, when he has to judge the relative merits of Andrews and Becker as candidates, Tom concludes that Andrews will be offered the position. But the disclosure of certain facts about Cortez in Scenario 2 alters Tom's evaluation of the relative merits of Andrews and Becker as candidates and as a consequence Tom concludes that Becker will be offered the position instead. Since the information Tom receives includes certain facts about Cortez, and since this information has been acquired from a reliable source (viz., the dean), Tom learns something important about the selection criterion used by the selection committee (viz., that expertise in metaphysics is not the only decisive criterion used by the selection committee). So we can say that Tom's epistemic choice from the menu represented by $a \vee b \vee c$ has epistemic relevance for Tom's epistemic decision and that Tom's epistemic choices are menu dependent.

Notice that the mere inclusion of facts about Cortez in the extended menu does not trigger the phenomenon of menu dependence. One needs to know in addition that the extra information has been acquired from a reliable source. The dean satisfies this requirement, but the example does not depend on the identity of the dean (as happens in many of the examples proposed by Sen). If, for example, the information were provided by a member

\footnotetext{
${ }^{25}$ In fact, as Rott points out in [Rot04], Aizerman's Axiom is also violated (see Section 1.2 for a statement of this condition). This coherence constraint corresponds to postulate $(* 8 c)$ via Rott's correspondence results [Rot01, pp. 197-198]. (Postulate (*8c) demands that if $\psi \in K * \varphi$, then $K * \varphi \subseteq K *(\varphi \wedge \psi)$.) This means that pseudo-rationalization is precluded (see footnote 5).
} 
of the selection committee the example would be equally effective in triggering a case of menu dependence. ${ }^{26}$

Here we want to consider two objections that one might raise to Example 3.4.1. The first objection is that we have not accurately represented the example. That is, one might object that we have not accurately represented the information that Tom receives from the dean. Allow us to illustrate this objection by way of example. On the one hand, in Scenario 2, when the dean informs Tom that either Andrews or Becker or Cortez will be offered the position $(a \vee b \vee c)$, one might contend that the dean's information at least leaves it open for Cortez to be offered the position. On the other hand, in Scenario 1, when then dean informs Tom that either Andrews or Becker will be offered the position $(a \vee b)$, the possibility that Cortez will be offered the position is seemingly excluded. So, one might say, we should have illustrated this difference in Scenario 1 by representing the dean's information by, say, $(a \vee b) \wedge \neg c$. Rott addresses this sort of objection in [Rot04]. Readers who are sympathetic with this objection should consult Rott's article.

The second objection is that we have conflated the notion of belief with expectation. ${ }^{27}$ One might contend that in Scenario 2, for example, Tom does not come to fully believe that Becker will get the position. Rather, Tom comes to fully believe, say, only that precisely one of Andrews, Becker, and Cortez will be offered the position and that Doyle will not be offered the position. Importantly, Tom only strongly expects that Becker will be offered the position. After all, if Tom were to fully believe that Becker will be offered the position, Tom would apparently be jumping to conclusions.

Indeed, expectations may be different from beliefs. And expectations may guide our beliefs without quite being part of them [GM94, p. 2]. Be this as it may, a principled distinction between expectations and beliefs has been quite elusive. In [Rot01], Rott writes:

We have not found a sharp boundary between beliefs and expectations. Any potential standard for separating beliefs from expectations may be contextually shifted, according to the situation one is facing. It is very doubtful whether an agent makes use of the same set of propositions in different situations-even if his doxastic state does not change at all. A loose conjecture may count as a full belief in party chat, but in the courtroom one ought to be firmly convinced of the truth of a proposition in order to affirm that one believes it to be true. Pragmatic considerations are needed to determine what qualifies as a belief (p. 29).

Gärdenfors and Makinson express a similar view in [GM94]:

Epistemologically, the difference between belief sets and expectations lies only in our attitude to them, i.e., what we are willing to do with them. For so long as we are using a belief set $K$, its elements function as full beliefs. But as soon as we seek to revise $K$, thus putting its elements into question, they lose the status of full belief and become merely expectations... (p. 35).

In this article, we will not attempt to explicate the distinction between beliefs and expectations. But we fully agree that Example 3.4.1 is most convincing if what is revised

\footnotetext{
${ }^{26}$ David Makinson suggested to us in a private communication that there might be cases of pure menu dependence, where the choice depends on the content of the menu, irrespective of the context in which it is offered. It is unclear whether there are pure cases of this sort. It seems difficult to find examples that do not depend on the reliability of the information source used to extend the menu. In any case, the distinction between pure and impure cases of menu dependence seems worthy of further analysis.

${ }^{27}$ The notion of expectation here should not be confused with the notion of expected utility in rational choice. Whereas expected utility concerns expectations of the values of various outcomes, here expectation concerns beliefs about the world (see [GM94, p. 5]). Perhaps the only dissenting voice regarding this point is Levi who in [Lev96] treats expectations as cognitive expected value. We use expectations here in the first epistemic sense of the word.
} 
are expectations rather than full beliefs. Thus, we may take $K$ in Example 3.4.1 to be the agent's expectations, without defeat, for this is a presumption often made in the literature of belief change (e.g., in [Spo], [PG96], [GM94]), and in particular, by Rott [Rot01]. This issue notwithstanding, the example still shows that postulate $(* 7)$ (and so condition $\alpha^{*}$ ) is violated, ${ }^{28}$ and the formal implications of these violations are what are at issue in this paper. ${ }^{29}$

So much for the first example. We now present an example that is structurally similar to the first example but where social norms play a crucial role.

3.4.2. Example. The candidates for a position in epistemology in a philosophy department are Anita Adams, John Becker, Peter Collins, Don Doyle, and Sasha Earl. Don Doyle is a star epistemologist, while John Becker is close in running. He is only slightly surpassed by Doyle with respect to objective merits. To be sure, Becker has a very good record of publications, and he is a candidate that gave one of the the best talks. Anita Adams is also a very good candidate. She happens to be African-American. Although she does excellent work, her work is not quite as good as Becker's work. Yet she is younger than Becker, and she is quite promising. Another candidate, Sasha Earl, is surpassed by Anita Adams in terms of objective merits. She is nonetheless a good candidate for the position. As it turns out, she also happens to be African-American.

Finally we have Peter Collins. Collins has done work of comparable quality to the work of Adams. But he is well-known for his political support of groups that promote white supremacy, and he is still involved in an unsettled case of harassment at a different university.

Now suppose Tom initially believes that neither Adams, Becker, Earl, nor Collins will be offered the position because he, like everyone else, believes that Doyle is the obvious candidate to be offered the position. Thus, Tom believes

$\neg a$ : Adams will not be offered the position.

$\neg b$ : Becker will not be offered the position.

$\neg c$ : Collins will not be offered the position.

$d$ : Doyle will be offered the position.

$\neg e$ : Earl will not be offered the position.

Tom ran into the dean while crossing campus, and they have decided to find a place to sit and chat. Tom takes the dean to be a very competent, serious, and honest man. Tom is also aware that the dean is the chairman of the selection committee.

Now consider the following scenarios:

Scenario 1. Tom learns from the dean that Adams, Becker, or Earl will be offered the position in epistemology $(a \vee b \vee e)$. Although Adams' work is surpassed by the work of Becker, she belongs to two demographic groups and is a better candidate

\footnotetext{
${ }^{28}$ Other supplementary postulates are violated as well, such as postulate $(* 8 c)$ (see footnote 25$)$. No basic postulates are violated in this example.

${ }^{29}$ Again, Levi traces a sharp distinction separating full beliefs and expectations. In [Lev96] he distinguishes between the ordinary versions of the postulates of belief change, like postulate $(* 7)$, and inductively extended versions of these postulates. And he has pointed out that the inductively extended version of postulate $(* 7)$ fails to hold. So, we assume that this would be his preferred explanation of examples like the one offered by Rott. Perhaps the distinction holds even if one does not buy Levi's theory of induction, and if one uses a different theory of induction instead. So the issue of what counts as a counterexample to well-known principles of belief formation sanctioned by AGM depends on a previous understanding of the notion of expectation as opposed to full belief. While Levi's strategy might work (assuming that one buys his notion of expectation) in the cases where menu dependence is the main mechanism, it is unclear whether this strategy applies to cases where the main underlying mechanism is determined by the use of social norms. More about this will be discussed below.
} 
for the position than Earl. Accordingly, Tom concludes that Adams will be offered the position.

Scenario 2. The dean informs Tom that Collins will be offered the position (c). Tom knows that the dean is an upright individual, so he does not doubt what the dean has told him.

Scenario 3. Tom learns from the dean that Adams, Becker, Collins, or Earl will be offered the position $(a \vee b \vee c \vee e)$. In this case the presence of Collins signals to Tom that the department might not take into account affirmative action. He concludes that the position will be offered to Becker.

The analysis of Tom's beliefs is similar to the previous example (among other things, we have $\neg b \in K *(a \vee b \vee e) \cap K * c$ and $\neg b \notin K *(a \vee b \vee c \vee e)$, so postulate $(* 7)$ is violated). But here we have the influence of a social norm in Scenario 1. Clearly in terms of considerations of objective merits alone Becker is expected to surpass Adams, Collins, and Earl. Nevertheless, when faced with the information given by $a \vee b \vee e$, that Becker will be offered the position $(b)$ is rendered unfeasible by a norm according to which, all things considered, candidates belonging to disadvantaged groups should be selected. Tom thereby concludes that Adams will be offered the position $(a)$. But when Tom is faced with the information given by $a \vee b \vee c \vee e$, we witness the phenomenon of menu dependence at work. Tom sees that the above social norm is not taken into account, and in this case Tom's belief change is guided by considerations of objective merits alone and so Tom concludes that Becker will be offered the position $(b)$.

Let us consider an analysis of this example in terms of possible worlds. We have five relevant worlds: $w_{1}=(\neg a, \neg b, \neg c, d, \neg e), w_{2}=(\neg a, b, \neg c, \neg d, \neg e), w_{3}=(a, \neg b, \neg c, \neg d, \neg e)$, $w_{4}=(\neg a, \neg b, c, \neg d, \neg e)$, and $w_{5}=(\neg a, \neg b, \neg c, \neg d, e) .{ }^{30}$ Given the background considerations of objective merits of the candidates, it is reasonable to suppose that we have an ordering $\geq$ over possible worlds which works as follows: $w_{1}$ dominates $w_{2}, w_{2}$ dominates $w_{3}$ and $w_{4}$, and $w_{3}$ is tied with $w_{4}$, while the latter tied worlds dominate $w_{5}$. Thus, where $\sim$ and $>$ are defined in the usual way ( $w \sim v$ :iff $w \geq v$ and $v \geq w ; w>v$ :iff $w \geq v$ and $v \nsupseteq w), w_{1}>w_{2}, w_{2}>w_{3}$ and $w_{2}>w_{4}, w_{3} \sim w_{4}$, and $w_{3}>w_{5}$ and $w_{4}>w_{5}$ (all other worlds are dominated by $w_{1}, w_{2}, w_{3}, w_{4}$, and $w_{5}$, and $w_{i} \geq w_{j}$ for $i \leq j$ ).

Now when $a \vee b \vee e$ is learned the underlying norm related to the promotion of underrepresented demographic groups makes the world $w_{2}$ unfeasible, while two worlds remain feasible, $w_{3}$ and $w_{5}$. Since Adams dominates Earl in terms of objective merits, Tom concludes that Adams will be offered the position $(a)$, whereby $w_{3}$ becomes admissible. Thus, if $\gamma$ is a semantic selection function that generates the revision function $*$, we have $\gamma(\llbracket a \vee b \vee e \rrbracket)=\left\{w_{3}\right\}$. But when the quadruple disjunction $a \vee b \vee c \vee e$ is learned the norm is seen to be playing no role. Considerations of objective merits alone lead Tom to conclude that Becker will be offered the position $(b)$. We accordingly have $\gamma(\llbracket a \vee b \vee c \vee e \rrbracket)=\left\{w_{2}\right\}$. Of course, since this example is an illustration of a violation of postulate $(* 7)$, there is no rational semantic selection function that models Tom's belief revision. Yet it seems reasonable to assume that the above ordering is relevant in this example.

Indeed, we can maintain the ordering $\geq$ over possible worlds, viewing the semantic selection function $\gamma$ that models Tom's belief revision as conditional upon a permissibility function $\pi$. When Tom learns $a \vee b \vee e$ in Scenario 1, the underlying social norm renders

\footnotetext{
${ }^{30}$ Here we adopt a notational convention: the expression $(a, \neg b, \neg c, \neg d, \neg e)$ denotes the possible world for which $a$ is true and the rest of the items $-b, c, e, d-$ are false.
} 
$w_{2}$ unfeasible while permitting $w_{3}$ and $w_{5}$ to remain feasible. We can thereby view the role of the permissibility function in such a way that $w_{2} \notin \pi(\llbracket a \vee b \vee e \rrbracket)$ and $\left\{w_{3}, w_{5}\right\} \subseteq$ $\pi(\llbracket a \vee b \vee e \rrbracket)$. If $\gamma(\llbracket a \vee b \vee e \rrbracket)$ is then understood to select those worlds optimal among those from $\pi(\llbracket a \vee b \vee e \rrbracket)$, then we have that $\gamma(\llbracket a \vee b \vee e \rrbracket)=\{w \in \pi(\llbracket a \vee b \vee e \rrbracket)$ : $w \geq w^{\prime}$ for all $\left.w^{\prime} \in \pi(\llbracket a \vee b \vee e \rrbracket)\right\}=\left\{w_{3}\right\}$. By contrast, when Tom learns $a \vee b \vee c \vee e$ in Scenario 3, the norm is seen to not be taken into account, so it is reasonable to take $\pi(\llbracket a \vee b \vee c \vee e \rrbracket)=\llbracket a \vee b \vee c \vee e \rrbracket$, whence $\gamma(\llbracket a \vee b \vee c \vee e \rrbracket)=\{w \in \pi(\llbracket a \vee b \vee c \vee e \rrbracket):$ $w \geq w^{\prime}$ for all $\left.w^{\prime} \in \pi(\llbracket a \vee b \vee c \vee e \rrbracket)\right\}=\left\{w_{2}\right\}$. In the next section we will return to this sort of analysis of belief change, introducing what we call norm-inclusive belief revision.

It is clear that there is an intimate connection between the underlying social norm relevant to the example and the expectations generated by it. Notice in the above example that although expectations are involved we do not have an inductive machinery (of the sort used by Levi in [Lev96]) to generate them. All epistemic choices are explained in terms of a unique ordering of epistemic options plus considerations of feasibility, which, in turn, are the consequence of the operation of underlying norms. ${ }^{31}$

Let us now consider another hiring example with a different structure. The example is simpler than the previous two examples considered above.

3.4.3. Example. Jeff Johns and Mara Lee Pearl are two outstanding candidates for a job search a philosophy department has been conducting. They are married, and Johns is a better candidate than Pearl all things considered. But Pearl is a decent candidate who could be a good addition to the department as a teacher. Johns has already another offer from an university in a different town, but, all things considered he would prefer an offer from this department. Tom, just like everybody else, believes that due to budget cuts the department will not be able to offer a position to either of them $(\neg j \wedge \neg p)$. Consider the following three scenarios:

Scenario 1. Tom is informed that Johns will be hired $(j)$. Under the point of view of merit and the convenience of the department the two states $(j \wedge \neg p)$ and $(j \wedge p)$ are tied, but Tom applies a norm in this case, whereby all things considered the unity of the family should be preserved. He concludes that both will be hired $(j \wedge p)$.

Scenario 2. Tom learns that Johns will not be hired but that Pearl will be hired $(\neg j \wedge p)$.

Scenario 3. Tom learns that either Johns will be hired or that Johns will not be hired but his wife will be hired. In this case Tom receives information that is compatible with a situation where the couple will have jobs in different towns $(\neg j \wedge p)$. The fact that this situation (which is the worst option for the merit ranking) is considered possible convinces Tom that the aforementioned norm does not apply here and by considerations of merit alone concludes that Johns will be hired $(j)$.

\footnotetext{
${ }^{31}$ A possible solution of compromise between Levi's inductive approach and the use of norms in examples of this sort could be to say that we are dealing with norms that induce or generate expectations. But in this case, these expectations do not seem to be those from a theory like Levi's but rather the purely epistemic expectations usually used in theories of belief change. Still, we can see a norm-sensitive operator of belief change as the composition of two operators: one classical AGM operator plus an inductive operator sanctioning an inductive jump made possible by the underlying norms. Most of what follows can be seen as a positive theory about this operator - where we provide new axioms that the operator should obey.
} 
So, we have that $p$ belong to the intersection of the first two revisions, but $p$ does not belong to the revision with the disjunction of the first two items.

An analysis in terms of possible worlds is possible here as well. The relevant worlds are $w_{1}=(j, p), w_{2}=(\neg j, \neg p), w_{3}=(j, \neg p)$ and $w_{4}=(\neg j, p)$. Under the point of view of merit and the convenience of the department worlds $w_{1}$ and $w_{3}$ are tied and optimal. The worst option in this ordering is $w_{4}$. When $j$ is learned the world $w_{3}$ is made unfeasible by an underlying norm promoting the unity of families whenever possible $\left(\pi\left(\left\{w_{1}, w_{3}\right\}\right)=\right.$ $\left.\left\{w_{1}\right\}\right)$. When the disjunction is learned in the third scenario the norm is deactivated and the agent settles in a theory corresponding to these three relevant worlds $\left(\pi\left(\left\{w_{1}, w_{2}, w_{3}\right\}\right)=\right.$ $\left.\left\{w_{1}, w_{2}, w_{3}\right\}\right)$. As with the previous examples, this represents a violation of postulates $(* 7)$ and condition $\alpha$.

At this point we expect to have convinced the reader that social norms are as ubiquitous in epistemology as they are in rational choice. It seems that there is a robust connection between the structure of our expectations and social norms. Social norms seem to justify many of our expectations and they seem crucial in the way we change them in the presence of new information.

To focus on a different type of social norm, let's consider social norms related to undergraduate students' beer drinking habits on the main campuses of American universities. Say that Tom is convinced that John is studying at home for an exam. Say as well that in this situation Tom learns that either John is at the local bar drinking beer with his friends or he is at the local bar drinking tea with his friends. Given this information, Tom would probably conclude that John is drinking beer with his friends. This expectation is formed by taking into account the norm that sanctions beer drinking habits in bars of this type among students. The norm plays a crucial role in the way one might form and change one's expectations.

Here we wish to emphasize that we are considering only social norms, not norms of another type (like legal norms). Social norms are the type of norms that Sen considers relevant in the realm of social choice. Our point here is that these norms are equally relevant in epistemology. Their main role is related to the process of forming and changing our expectations.

In this section we have offered examples in which postulate $(* 7)$ (among many other postulates) is violated. Yet the belief changes involved in these examples seem perfectly reasonable. It is indeed evident that some violations of postulate $(* 7)$ can be explained in terms of the influence of social norms in belief formation. Our goal in the remainder of this article is to develop a theory of belief revision which takes into account the role social norms play in belief formation.

\section{PART IV: Norm-InClusive Belief REVISION}

In Section 3.3, we reviewed Hans Rott's correspondence results for belief revision, which show how postulates of belief revision correspond in a one-to-one fashion to coherence constraints of rational choice. We saw how these results, furnished within a general framework, bear upon rationalizability in belief revision.

In Section 3.4, we encountered several counterexamples to postulates of belief revisionin particular, to postulate $(* 7)$. In virtue of Rott's correspondence results, these counterexamples threatened the all but universal presumption that belief revision is relational. We argued that many counterexamples which threaten this presumption are driven by the influence social norms have in belief formation. We have reviewed recent work concerned 
with accommodating the role social norms play in choice. We have also improved upon the results of this work, developing a theory of norm-conditional choice.

The primary purpose of this section is to introduce a new theory of belief revision, called norm-inclusive belief revision. Like the theory of norm-conditional choice we discussed earlier, norm-inclusive belief revision is intended to take into account the role social norms play in belief change. Also like the theory of norm-conditional choice, norm-inclusive belief revision is an extension of the classical belief revision theory investigated by many researchers in formal epistemology.

In this section we will introduce postulates of norm-inclusive belief revision. We will then state and prove correspondence theorems for norm-inclusive belief revision, providing a direct connection between conditions imposed upon norm-conditional choice models and conditions placed upon what we call norm-inclusive revision models. We will conclude with discussion and an example that illustrates our theory at work.

4.1. Postulates. Roughly, a norm-inclusive belief revision model for a belief set $K$ is a

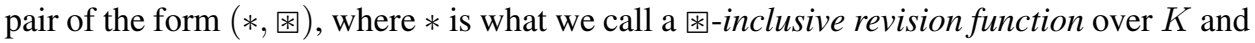
困 is what we call a norm representation function for $*$. Intuitively, $K * \varphi$ is the revision of $K$ that is compatible with those beliefs $K \circledast \varphi$ an underlying social norm warrants for acceptance when $\varphi$ is learned. ${ }^{32}$

As with standard theories of belief revision, we presume that $*$ satisfies postulates $(* 1)$, $(* 2)$, and $(* 6)$. We also presume $*$ satisfies the following postulates:

(困1) $K$ 困 $\varphi=\mathrm{Cn}(K$ 困 $\varphi)$.

(Normative Closure)

(困2) $\varphi \in K$ 困 $\varphi$.

(Normative Success)

(困6) If $\operatorname{Cn}(\{\varphi\})=\operatorname{Cn}(\{\psi\})$, then $K$ 困 $\varphi=K$ 国 $\psi$. (Normative Extensionality)

Postulate (困1) simply requires that the set of beliefs an underlying social norm warrants for acceptance is closed under logical consequence. According to postulate (因2), even when a social norm is operative in a revision, a norm representation function must give the incoming information priority. Postulate (困6) demands that a norm representation function treats logically equivalent information in the same way. In particular, a norm representation function is not sensitive to the linguistic formulation of incoming information.

With this we can offer a precise formulation of what we mean by a norm-conditional belief revision model.

Definition 4.1. Let $K$ be a belief set, let $*$ be a function satisfying $(* 1),(* 2)$, and $(* 6)$, and let $⿴$ be a function satisfying (困1), (因2), and (困6). We call the pair $(*$, 目) a norminclusive revision model over $K$ if for every $\varphi \in \operatorname{For}(\mathcal{L}), K \Theta \varphi \subseteq K * \varphi{ }^{33}$

Thus, we require that a norm-inclusive belief revision model satisfies the following postulate:

(*[困]) $K$ 目 $\varphi \subseteq K * \varphi . \quad$ (Norm-Inclusive Revision)

As indicated above, the intuition here is that for an agent revising its beliefs $K$ by a sentence $\varphi, K$ 東 $\varphi$ represents those beliefs that an underlying social norm warrants for acceptance when $\varphi$ is learned. Thus, postulate $(*[$ 困] ) signifies that an agent ought to believe every sentence $\psi$ an underlying norm warrants for acceptance in the revision of $K$ by $\varphi$.

\footnotetext{
${ }^{32} \mathrm{~A}$ norm warrants a belief $\psi$ for acceptance if the norm makes the acceptance of $\psi$ permissible. The warranted beliefs are the beliefs permitted by the norm.

${ }^{33}$ Of course, it would have been sufficient to specify that $*$ satisfies only postulates $(* 2)$ and $(* 6)$, without specifying that $*$ satisfies $(* 1)$.
} 
The following postulates are analogues of postulates $(* 3),(* 4)$, and $(* 5)$ :

(困3) $K$ 困 $\varphi \subseteq \mathrm{Cn}(K \cup\{\varphi\})$.

(困4) If $\neg \varphi \notin K$, then $\operatorname{Cn}(K \cup\{\varphi\}) \subseteq K$ 困 $\varphi$.

Clearly, a $*$-inclusive revision function $*$ over $K$ satisfies postulate $(* 3)$ only if $*$ satisfies postulate (困3), and $*$ satisfies postulate $(* 4)$ provided $*$ satisfies postulate $($ 困 4$)$. We stress that a norm representation function $\circledast$ need not represent a belief change operation, so one might find it undesirable to impose postulate (因3) and especially postulate (因4). Yet if $*$ is intended to represent an AGM-style belief revision operation, the following postulate seems to be a reasonable constraint for norm representation functions:

(困, ${ }_{\iota, K}$ ) If $\neg \varphi \notin K$, then $K$ 困 $\varphi \subseteq \mathrm{Cn}(\{\varphi\})$. (Norm in Absentia with respect to $K$ )

According to postulate $\left(\mathbb{*}_{\iota, K}\right)$, a norm representation function may modulate incoming information only when the information is incompatible with an agent's beliefs. Thus, if a sentence $\varphi$ is consistent with a belief set $K$, then the beliefs warranted for acceptance by the underlying norm should not go beyond the information from $\operatorname{Cn}(\{\varphi\})$. In conjunction with postulates $(* 3)$ and $(* 4)$, this means that revision by a sentence compatible with an agent's beliefs proceeds purely by expansion.

The following postulate corresponds to postulate $(* 5)$.

(困5) If $\operatorname{Cn}(\{\varphi\}) \neq \operatorname{For}(\mathcal{L})$, then $K$ 国 $\varphi \neq \operatorname{For}(\mathcal{L})$.

A relative of postulate (困5), the next mixed postulate demands that if the set of beliefs an underlying norm warrants for acceptance in the revision of a belief set $K$ by sentence $\varphi$ is consistent, then the revision of $K$ by $\varphi$ ought to be consistent as well.

\section{$(*[$ 困 $] 5) \quad$ If $K$ 类 $\varphi \neq \operatorname{For}(\mathcal{L})$, then $K * \varphi \neq \operatorname{For}(\mathcal{L}) . \quad$ (Norm-Inclusive Consistency)}

It is an easy matter to check that if a $*$-inclusive revision function $*$ over $K$ satisfies postulate $(* 5)$, then the norm representation function 因 satisfies postulate $($ 困5) while the norm-inclusive revision model $(*$, 国) satisfies postulate $(*[$ 国]5). Indeed, if $(*$, $*)$ is a

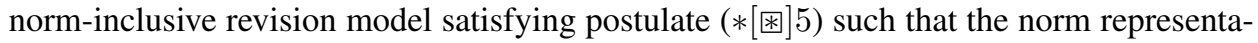
tion function 娄 satisfies postulate (困5), then the norm-inclusive revision function $*$ satisfies postulate $(* 5)$.

As with condition $\pi_{\iota}$, the next postulate represents an interesting limit case.

(因 $九) \quad K$ 因 $\varphi \subseteq \operatorname{Cn}(\{\varphi\})$. (Norm in Absentia)

Postulate $\left(\right.$ 困 $\left._{\iota}\right)$ signifies that the underlying norm in question does not sanction the accep-

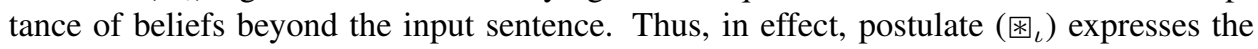
fact that the underlying norm, even if it is operative, does not have any real influence on belief formation.

We finally turn to the central postulate of this section.

$$
\begin{aligned}
& \left(*[\text { [ }] \mathrm{R}_{\infty}\right) \quad \text { For every non-empty } I \subseteq \operatorname{For}(\mathcal{L}) \text {., } \\
& \text { (Norm-Inclusive Revision Coherence) } \\
& \text { if } \bigcap_{\psi \in I} K \circledast \psi \subseteq K \circledast \varphi \text {, then } K * \varphi \subseteq \mathrm{Cn}\left(\left(\bigcup_{\psi \in I} K * \psi\right) \cup K \circledast \varphi\right) \text {. }
\end{aligned}
$$

This postulate, as it presently stands, appears to be quite complicated. As with condition $\gamma[\pi] \mathbf{R}_{\infty}$, the reason for this is that we have not placed any substantial conditions upon norm representation functions. Nonetheless, the proof of the pudding is in the eating. Our goal here is to show how it is possible to accommodate the influence of social norms in belief revision. As we will see, postulate $\left(*[\circledast] \mathrm{R}_{\infty}\right)$ embodies the minimal commitments one must undertake for rational norm-inclusive belief revision. 
4.2. Correspondence Theorems. In this subsection we will present correspondence theorems connecting postulates of norm-inclusive belief revision to coherence constraints of norm-conditional choice. As in Section 3.3, we begin with several definitions.

Definition 4.2. A semantic norm-conditional choice model is a norm-conditional choice model on choice space $\left(\mathcal{W}_{\mathcal{L}}, \mathcal{E}_{\mathcal{L}}\right)$.

In Section 3.3, we reviewed the notion of a choice-based revision function. We now introduce the notion of a norm-inclusive choice-based revision model.

Definition 4.3. Let $K$ be a belief set, and let $(\gamma, \pi)$ be a semantic norm-conditional choice model. The semantic norm-inclusive choice-based revision model $(*$, *) over K generated by $(\gamma, \pi)$ is defined by setting for every $\varphi \in \operatorname{For}(\mathcal{L})$,

$$
K * \varphi:= \begin{cases}\widehat{\gamma(\llbracket \varphi \rrbracket)} & \text { if } \gamma(\llbracket \varphi \rrbracket) \neq \emptyset \\ \operatorname{For}(\mathcal{L}) & \text { otherwise }\end{cases}
$$

and

$$
K \circledast \varphi:= \begin{cases}\widehat{\pi(\llbracket \varphi \rrbracket)} & \text { if } \pi(\llbracket \varphi \rrbracket) \neq \emptyset \\ \operatorname{For}(\mathcal{L}) & \text { otherwise. }\end{cases}
$$

We say that $(\gamma, \pi)$ generates $(*$, 困) or that $(*$, 困) is generated by $(\gamma, \pi)$.

Observe that every norm-inclusive choice-based revision model $(*$, 困) over $K$ is such that $*$ satisfies postulates $(* 1),(* 2)$, and $(* 6)$, 圈 satisfies postulates (因), (因), and (因6), and $(*$, 困) satisfies postulate $(*$ 困). Thus, a norm-inclusive choice-based revision model is indeed a norm-inclusive revision model. As we will see below, the converse holds as well.

The intuitive interpretation of $K * \varphi$ in this context is similar to that of the usual interpretation: An agent believes a sentence $\psi$ in the revision of $K$ by $\varphi$ just in case $\psi$ is true in all the most 'plausible' $\pi$-permissible worlds in which $\varphi$ is true.

We now introduce a final coherence constraint for norm-conditional choice models. As with conditions $\left(\mathrm{F} 1_{B}\right)$ and $\left(\mathrm{F} 2_{B}\right)$, this coherence constraint is relative to a set $B$ of options.

$\left(\pi_{\iota, B}\right) \quad$ For every $S \in \mathscr{S}$, if $S \cap B \neq \emptyset$, then $S \subseteq \pi(S)$. (Norm in Absentia with respect to $B$ )

Shortly we will see that this condition corresponds to postulate $\left(\Theta_{\iota, B}\right)$ of norm-inclusive belief revision.

We offer a final definition.

Definition 4.4. Let $K$ be a belief set. We call $(*, \circledast)$ a (regular, norm-conditional regular, rational, G-rational) norm-inclusive choice-based revision model over $K$ if there is a (regular, $\pi$-conditional regular, $\pi$-rational, $\mathrm{G} \pi$-rational) semantic norm-conditional choice model $(\gamma, \pi)$ that generates $(*$, 困).

We are now in a position to state and prove the long-awaited correspondence theorems.

Theorem 4.5. Let $K$ be a belief set. For every semantic norm-conditional choice model $(\gamma, \pi)$ which satisfies 


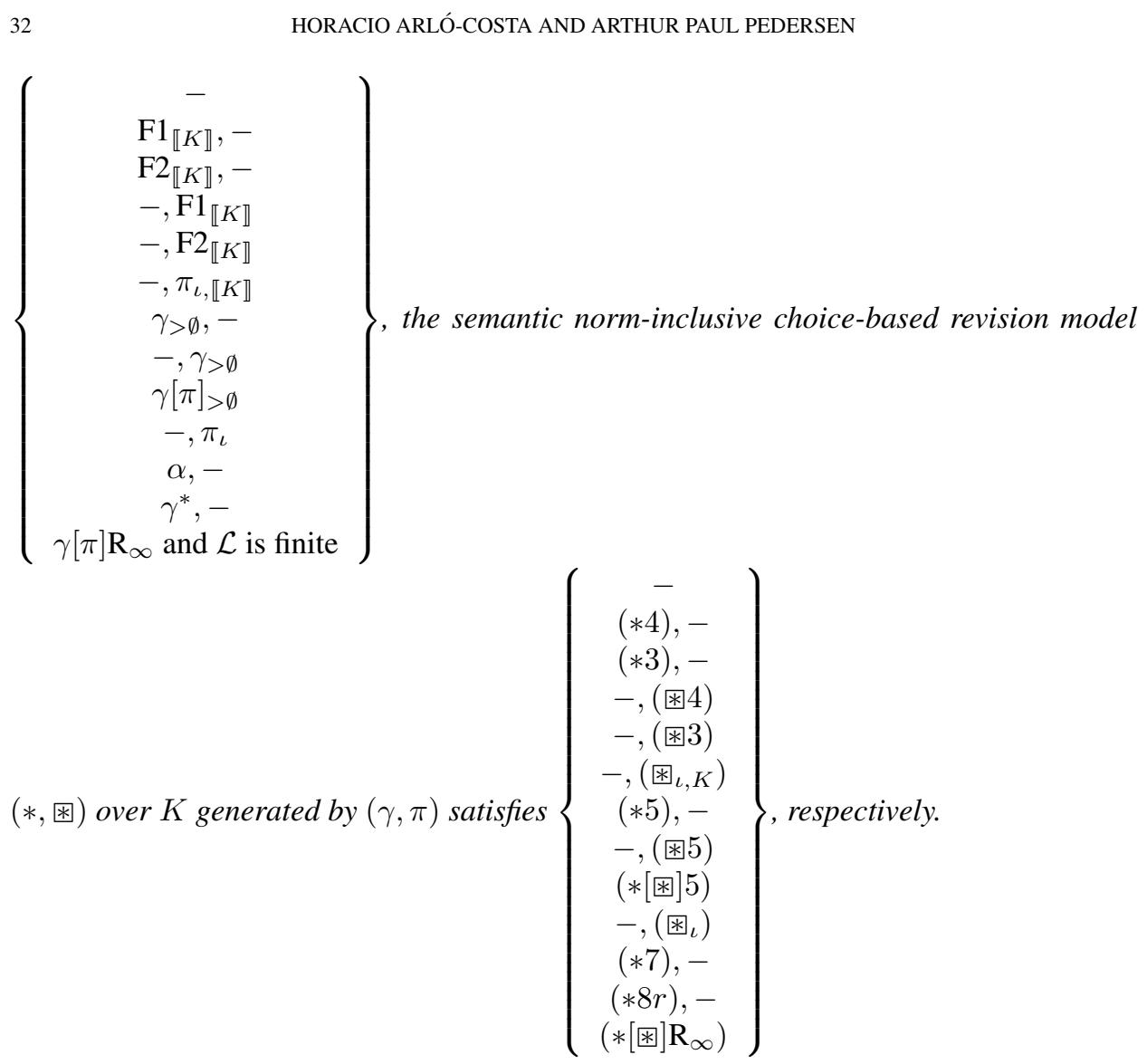

Proof. Let $(\gamma, \pi)$ be a semantic norm-conditional choice model, and let $(*$, 困) be the semantic norm-inclusive choice-based revision model over $K$ generated by $(\gamma, \pi)$. We have seen above that $(*$, 困) is indeed a norm-inclusive revision model over $K$. We only prove the implications for a subset of the conditions of the theorem, leaving the remaining implications to the reader.

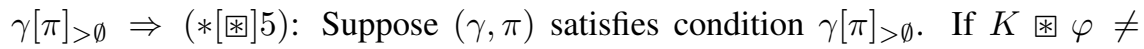
$\operatorname{For}(\mathcal{L})$, then by definition $\pi(\llbracket \varphi \rrbracket) \neq \emptyset$, so by condition $\gamma[\pi]_{>\emptyset}$, we have that $\gamma(\llbracket \varphi \rrbracket) \neq \emptyset$, whence again by definition $K * \varphi \neq \operatorname{For}(\mathcal{L})$.

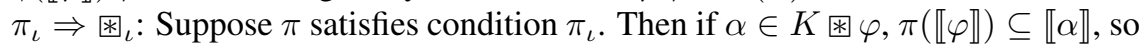
by condition $\pi_{\iota}$ it follows that $\llbracket \operatorname{Cn}(\{\varphi\}) \rrbracket=\llbracket \varphi \rrbracket \subseteq \llbracket \alpha \rrbracket$, whereby $\alpha \in \operatorname{Cn}(\{\varphi\})$, as desired.

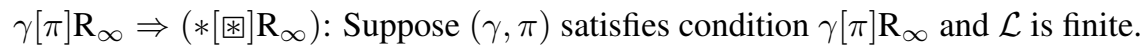
Let $I \subseteq \operatorname{For}(\mathcal{L})$ be such that $I \neq \emptyset$. Suppose $\bigcap_{\psi \in I} K \Theta \psi \subseteq K$ 困 $\varphi$. Then since $\mathcal{L}$ is finite, it follows that $\llbracket K$ 田 $\varphi \rrbracket \subseteq \llbracket \bigcap_{\psi \in I} K$ 困 $\psi \rrbracket=\bigcup_{\psi \in I} \llbracket K$ 困 $\psi \rrbracket$. Again, since $\mathcal{L}$ is finite, $\pi$ is complete, so $\pi(\llbracket \varphi \rrbracket) \subseteq \bigcup_{\psi \in I} \pi(\llbracket \psi \rrbracket)$, whence it follows by condition $\gamma[\pi] \mathbf{R}_{\infty}$ that $\pi(\llbracket \varphi \rrbracket) \cap\left(\bigcap_{\psi \in I} \gamma(\llbracket \psi \rrbracket)\right) \subseteq \gamma(\llbracket \varphi \rrbracket)$. Now because $\gamma$ and $\pi$ 
are complete,

$$
\begin{aligned}
\llbracket \operatorname{Cn}\left(K \circledast \varphi \cup\left(\bigcup_{\psi \in I} K * \psi\right)\right) \rrbracket & =\llbracket K \circledast \varphi \cup\left(\bigcup_{\psi \in I} K * \psi\right) \rrbracket \\
& =\llbracket K \otimes \varphi \rrbracket \cap \llbracket \bigcup_{\psi \in I} K * \psi \rrbracket \\
& =\llbracket K \circledast \varphi \rrbracket \cap\left(\bigcap_{\psi \in I} \llbracket K * \psi \rrbracket\right) \\
& =\pi(\llbracket \varphi \rrbracket) \cap\left(\bigcap_{\psi \in I} \gamma(\llbracket \psi \rrbracket)\right) \subseteq \gamma(\llbracket \varphi \rrbracket)=\llbracket K * \varphi \rrbracket .
\end{aligned}
$$

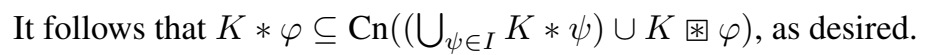

Theorem 4.6. Every norm-inclusive revision model (*, 困) over a belief set $K$ which satisfies

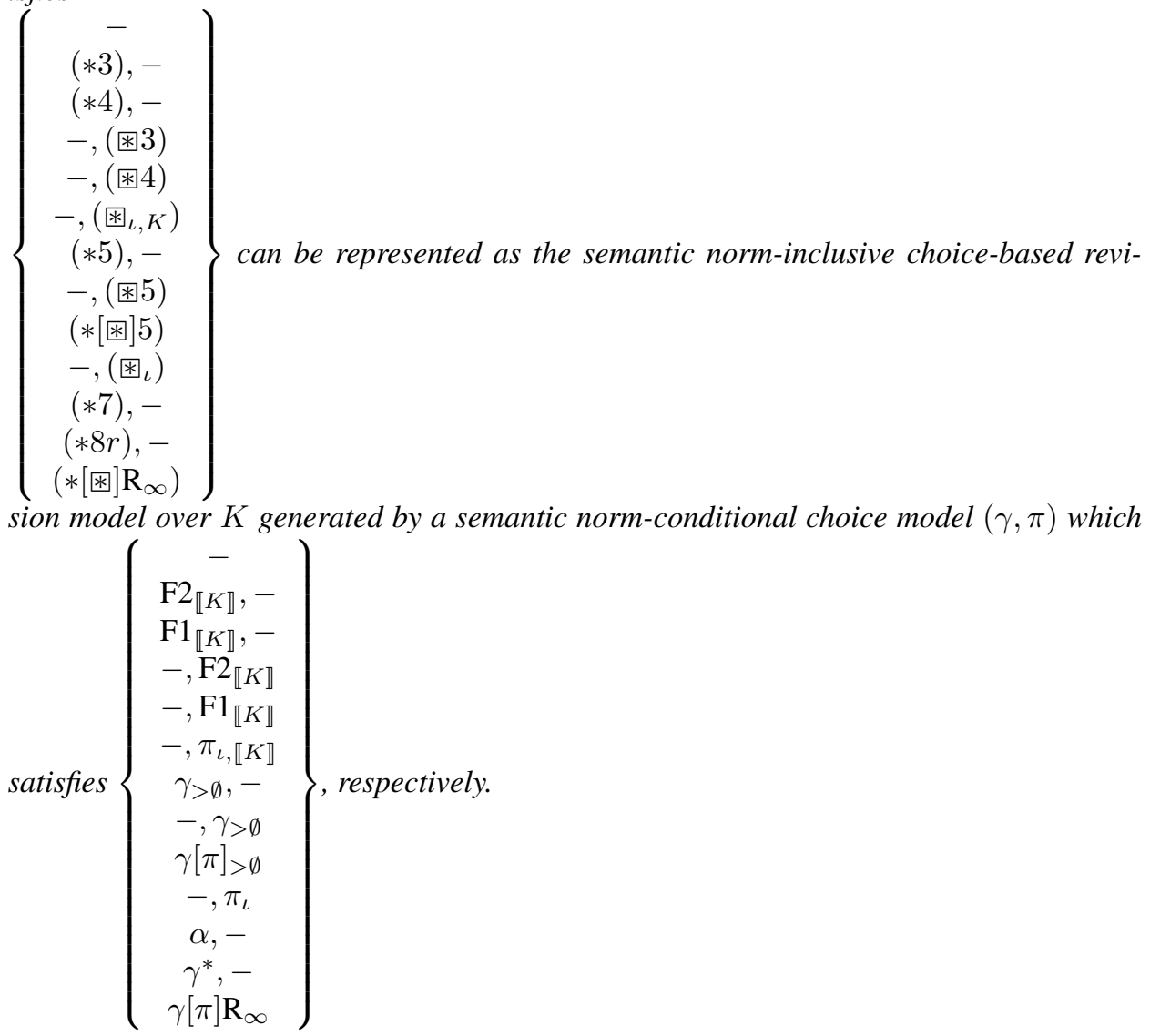

Proof. Let $(*$, 困) be a norm-inclusive revision model over a belief set $K$. We define a semantic norm-conditional choice model $(\gamma, \pi)$ by setting for every $\varphi \in \operatorname{For}(\mathcal{L}), \gamma(\llbracket \varphi \rrbracket):=$ $\llbracket K * \varphi \rrbracket$ and $\pi(\llbracket \varphi \rrbracket):=\llbracket K$ 目 $\varphi \rrbracket$. Because by definition $*$ satisfies postulate $(* 1),(* 2)$, and $(* 6)$, 困 satisfies postulates (困1), (困2), and (因6), and $(*$, 因) satisfies postulate $(*[$ 困]), clearly $(\gamma, \pi)$ is a semantic norm-conditional choice model. 
We must first show that $(\gamma, \pi)$ generates $(*$, 因). Let $\varphi \in \operatorname{For}(\mathcal{L})$. On the one hand, if $\gamma(\llbracket \varphi \rrbracket)=\emptyset$, then $\llbracket K * \varphi \rrbracket=\emptyset$, so $K * \varphi=\operatorname{For}(\mathcal{L})$. On the other hand, if $\gamma(\llbracket \varphi \rrbracket) \neq \emptyset$, then $K * \varphi=\llbracket \widehat{K * \varphi \rrbracket}=\widehat{\gamma(\llbracket \varphi \rrbracket)}$. We have thereby shown that

$$
K * \varphi= \begin{cases}\widehat{\gamma(\llbracket \varphi \rrbracket)} & \text { if } \gamma(\llbracket \varphi \rrbracket) \neq \emptyset \\ \operatorname{For}(\mathcal{L}) & \text { otherwise }\end{cases}
$$

A similar argument shows that

$$
K \text { 困 } \varphi= \begin{cases}\widehat{\pi(\llbracket \varphi \rrbracket)} & \text { if } \pi(\llbracket \varphi \rrbracket) \neq \emptyset \\ \operatorname{For}(\mathcal{L}) & \text { otherwise. }\end{cases}
$$

Hence, $(\gamma, \pi)$ generates $(*$, 困). We now turn to prove the implications of the theorem. As before, we only prove the implications for a subset of the postulates of the theorem.

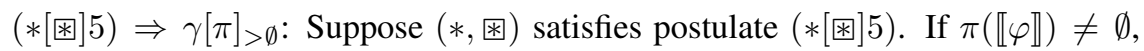
then $\llbracket K$ $⿴ 囗 ⿱ 一 一) \neq \emptyset$ and so $K$ 困 $\varphi \neq \operatorname{For}(\mathcal{L})$, whence by postulate $(*[$ 困]5) it follows that $K * \varphi \neq \operatorname{For}(\mathcal{L})$ and therefore $\gamma(\llbracket \varphi \rrbracket)=\llbracket K * \varphi \rrbracket \neq \emptyset$, as desired.

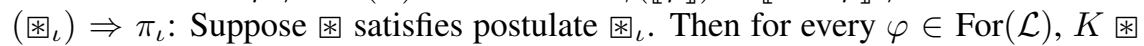
$\varphi \subseteq \operatorname{Cn}(\{\varphi\})$ and so $\llbracket \varphi \rrbracket=\llbracket \operatorname{Cn}(\{\varphi\}) \rrbracket \subseteq \llbracket K ⿴ \varphi \rrbracket=\pi(\llbracket \varphi \rrbracket)$.

$\left(*[\right.$ 困 $\left.] \mathbf{R}_{\infty}\right) \Rightarrow \gamma[\pi] \mathbf{R}_{\infty}$ : Suppose $\left(*\right.$, 国) satisfies postulate $\left(*\left[\right.\right.$ 国] $\left.\mathbf{R}_{\infty}\right)$. Let $I \subseteq \mathcal{E}_{\mathcal{L}}$ be such that $I \neq \emptyset$. Suppose $\pi(\llbracket \varphi \rrbracket) \subseteq \bigcup_{\llbracket \psi \rrbracket \in I} \pi(\llbracket \psi \rrbracket)$. Then $\llbracket K$ 娄 $\varphi \rrbracket \subseteq$

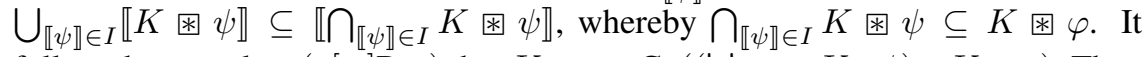
follows by postulate $\left(*[\right.$ [ $\left.] \mathrm{R}_{\infty}\right)$ that $K * \varphi \subseteq \mathrm{Cn}\left(\left(\bigcup_{\llbracket \psi] \in I} K * \psi\right) \cup K\right.$ 国 $)$. Then

$$
\begin{aligned}
\pi(\llbracket \varphi \rrbracket) \cap\left(\bigcap_{\llbracket \psi \rrbracket \in I} \gamma(\llbracket \psi \rrbracket)\right) & =\llbracket K \boxplus \varphi \rrbracket \cap\left(\bigcap_{\llbracket \psi \rrbracket \in I} \llbracket K * \psi \rrbracket\right) \\
& =\llbracket K ⿴ \varphi \rrbracket \cap \llbracket \bigcup_{\llbracket \psi \rrbracket \in I} K * \psi \rrbracket \\
& =\llbracket K \circledast \varphi \cup\left(\bigcup_{\llbracket \psi \rrbracket \in I} K * \psi\right) \rrbracket \\
& =\llbracket \operatorname{Cn}\left(K \boxplus \varphi \cup\left(\bigcup_{\llbracket \psi \rrbracket \in I} K * \psi\right)\right) \rrbracket \subseteq \llbracket K * \varphi \rrbracket=\gamma(\llbracket \varphi \rrbracket) .
\end{aligned}
$$

That is, $\pi(\llbracket \varphi \rrbracket) \cap\left(\bigcap_{\llbracket \psi \rrbracket \in I} \gamma(\llbracket \psi \rrbracket)\right) \subseteq \gamma(\llbracket \varphi \rrbracket)$, as desired.

In light of the results of Section 2.2, we have the following corollary.

Corollary 4.7. Let $\mathcal{L}$ be finite, and let $K$ be a belief set.

(i) A pair $(*, \circledast)$ is a rational norm-inclusive choice-based revision model over $K$ if and only if it is a norm-inclusive revision model satisfying postulate $\left(*\left[{ }^{*}\right] \mathbf{R}_{\infty}\right)$.

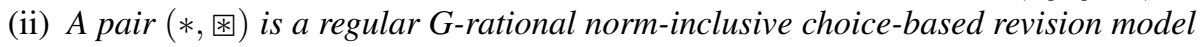
over $K$ if and only if it is a norm-inclusive revision model satisfying postulates $(* 5)$ and $\left(*[\right.$ 困 $\left.] \mathrm{R}_{\infty}\right)$.

(iii) A pair (*, 因) is a norm-conditional regular G-rational norm-inclusive choicebased revision model over $K$ if and only if it is a norm-inclusive revision model satisfying postulates $\left(*\left[\mathbb{*}^{\prime}\right] 5\right)$ and $\left(*[{ }] \mathrm{R}_{\infty}\right)$. 


\section{EXAMPLES AND DISCUSSION}

The modular nature of the correspondence results of the previous section afford applicability to a variety of theories of belief revision. A theorist of belief revision can utilize our semantic representation of norm-inclusive belief revision to accommodate the influence of social norms in belief formation. Indeed, because our framework extends the classical framework in which belief revision is studied, a theorist can utilize our framework even in cases for which social norms do not have any real influence on belief formation. ${ }^{34}$ Of course, a significant benefit of adopting our framework is that it offers a way to study belief revision with an eye toward the possibility that social norms sway belief formation.

The minimal conditions required for rational norm-inclusive belief revision are embodied in Corollary 4.7. We accordingly take the postulates comprising this corollary to represent the central conditions for norm-inclusive belief revision. As we mentioned earlier, we have not imposed any substantial conditions on the behavior of norm representation functions. We invite belief revision theorists to investigate what conditions can be plausibly imposed on norm representation functions.

As the reader may suspect, the above correspondence results translate naturally to correspondence results for what might be called norm-inclusive non-monotonic reasoning. Thus, our correspondence results also afford applicability to a variety of theories of nonmonotonic reasoning. We take this to be a virtue of the modular nature of our results.

We now illustrate our theory at work. We continue with Example 3.4.2 of Section 3.4.

5.1. Example. Recall the analysis of Example 3.4.2 in terms of possible worlds. We have five relevant worlds: $:^{35}$

$$
\begin{aligned}
& w_{1}=(\neg a, \neg b, \neg c, d, \neg e) \\
& w_{2}=(\neg a, b, \neg c, \neg d, \neg e) \\
& w_{3}=(a, \neg b, \neg c, \neg d, \neg e) \\
& w_{4}=(\neg a, \neg b, c, \neg d, \neg e) \\
& w_{5}=(\neg a, \neg b, \neg c, \neg d, e) .
\end{aligned}
$$

The ordering $\geq$ over possible worlds is such that $w_{1}>w_{2}, w_{2}>w_{3}$ and $w_{2}>w_{4}$, $w_{3} \sim w_{4}$, and $w_{3}>w_{5}$ and $w_{4}>w_{5}$ (all other worlds are dominated by $w_{1}, w_{2}, w_{3}, w_{4}$, and $w_{5}$, and $w_{i} \geq w_{j}$ for $i \leq j$ ).

We may first inquire about the contents of $K \circledast(a \vee b \vee e)$, where $K$ is the theory that corresponds to $w_{1}$. Here we have a norm related to the promotion of underrepresented demographic groups. According to the norm, $w_{2}$ is unfeasible, while two worlds will remain feasible, $w_{3}$ and $w_{5}$. So in terms of a norm representation function, if we take $\pi(\llbracket a \vee b \vee e \rrbracket)=\left\{w_{3}, w_{5}\right\}$, we will have that $K$ 困 $(a \vee b \vee e)$ is the intersection of $w_{3}$ and $w_{5}$-namely, $\operatorname{Cn}(\{a \vee e, \neg(a \wedge e), \neg b, \neg c, \neg d\})$.

We now inquire about the contents of $K *(a \vee b \vee e)$. In this case the idea is to pick the worlds that are optimal among the worlds that are normatively feasible. In terms of a $\pi$-conditional semantic selection function $\gamma$, we have $\gamma(\llbracket a \vee b \vee e \rrbracket)=\{w \in \pi(\llbracket a \vee b \vee e \rrbracket)$ : $w \geq w^{\prime}$ for all $\left.w^{\prime} \in \pi(\llbracket a \vee b \vee e \rrbracket)\right\}=\left\{w_{3}\right\}$. So the theory that corresponds to $w_{3}$ gives us $\bar{K} *(a \vee b \vee e)$.

We conclude this section with an intriguing variant of the example we presented above.

\footnotetext{
${ }^{34}$ When social norms do not have any real on influence belief formation, postulate $\circledast_{\iota}$ is satisfied.

${ }^{35}$ As before, we adopt the notational convention that $(a, \neg b, \neg c, \neg d, \neg e)$ denotes the possible world for which $a$ is true and the rest of the items $-b, c, e, d-$ are false.
} 
5.2. Example. Consider Example 3.4.2 of Section 3.4, but suppose instead that Tom is initially in suspense about who will be hired. Now rerun the scenarios considered in Example 3.4.2. The example so constructed preserves the format of Example 3.4.2, but now the information given by the Dean is compatible with Tom's initial beliefs. As with the previous examples, this example illustrates a violation of postulate $(* 7)$ (among other things). But here another postulate is violated. In the first scenario, for example, postulate $(* 3)$ is violated. Although the information Tom learns is compatible with his view, he does not change his view by expansion. Yet in the previous examples the changes in each scenario are AGM-permissible changes insofar as they satisfy postulate $(* 3)$.

One possible reaction to this variant of Example 3.4.2 is to say that Rott's examples and some of its possible variations do not really reveal violations of the AGM postulates but violations of inductively extended versions of these postulates (this seems to be Levi's position regarding the original examples - see [Lev96] for a precise definition of inductive expansion). So in the case of the change with $(a \vee b \vee e)$, the agent first accepts the disjunction in a way compatible with AGM and then jumps to conclusions using some inductive method.

Our theory also offers an explanation of these examples. In the first scenario, for example, a norm related to the promotion of unrepresented minorities is seen to be active and so two salient worlds remain feasible (the world where Adams is selected and nobody else is selected and the world where Earl is selected and nobody else is selected). One then optimizes over these feasible worlds, ultimately selecting Adam's world $\left(w_{3}\right)$. So as long as we take the previous example seriously, perhaps we have no reason to endorse postulate $(* 3)$ in a theory of norm-inclusive belief change. We do not, nevertheless, exclude the possibility that there could be different applications of our formalism where $*$ obeys all the basic AGM postulates.

The main issue analyzed in this paper (how to extend rationalizability in order to deal with cases where social norms are relevant) is independent of the considerations about the role of postulate $(* 3)$. Our representation results and our correspondence results are modular, permitting a formal separation of these issues.

\section{CONCLUSION AND Future WORK}

The classical theory of rational choice deriving from the work of mathematical economists such as Arrow, Richter, Samuelson, and Sen has important connections with the theory of belief change initiated by the seminal work of Alchourrón, Gärdenfors, and Makinson [AGM85]. Rott has articulated the formal and conceptual consequences of this connection in his recent [Rot01]. Usually when this connection is studied the theory of choice is taken as the more primitive and secure theory, from which several consequences for the theory of belief revision are drawn. But rarely are results in the theory of belief revision applied to produce novel results in the theory of choice.

One of the first theorems offered in our article is nevertheless of this type (see Theorem 1.6). Indeed, in Section 1.2 we undertook the traditional problem of stating necessary and sufficient conditions for G-rationalizability, whereupon we furnished a complete characterization of G-rationalizability in terms of a coherence constraint (viz., condition $\left.\gamma \mathbf{R}_{\infty}\right)^{36}$ that is a generalization of a condition considered by AGM in [AGM85]. This condition permits a characterization of G-rationality that stands independently of constraints on the underlying domain. Consequently, this condition permits an extension of the interesting

\footnotetext{
${ }^{36}$ Condition $\gamma_{1>\emptyset}$ should be imposed as well, but the central condition in the result is $\gamma \mathrm{R}_{\infty}$.
} 
program concerned with the foundations of rational choice, as investigated by mathematical economists such as Richter [Ric66, Ric71].

The usual restrictions on domains required by traditional results for rationalizability (of the sort Sen offers in [Sen71]) are incompatible with the empirical applicability of the theory of rational choice, something many economists nevertheless consider desirable. Thus, only a theory of rational choice formulated in terms of what Suzumura calls general domains (see [Suz83, p. 17]) should be considered acceptable for an empirically viable theory of choice. Our characterization of G-rationalizability offers a result of this type, with $\gamma \mathbf{R}_{\infty}$ playing an essential role.

The first half of this article focused on a problem for the standard theory of rational choice, a problem Amartya Sen has given careful attention in a series of important articles. The problem is associated with the role of social norms in choice. Sen has presented various examples which illustrate that the influence of social norms in choice threatens a central idea in the theory of rational choice - that choice functions are rationalizable. In particular, Sen has offered several examples which represent violations of coherence constraints such as condition $\alpha$ in cases where norms are operative. The central challenge prompted by Sen's examples (which he left unresolved in his articles on this issue) is whether it is possible to extend the theory of choice to accommodate the role of social norms in rational choice.

The first step needed to extend the existing theory of choice is to develop a formal framework that gives footing to social norms. There is a rich literature focusing on the role of social norms in choice from the seminal paper by Elster [Els89b] to the more recent and ambitious work by Cristina Bicchieri [Bic06]. Much of this work focuses on providing rational reconstructions of social norms (this is so especially in the philosophical literature). As an illustration, in the case of Bicchieri the idea is to provide epistemic conditions (in terms of expectations and conditional expectations) which are necessary and sufficient for the very existence of social norms. To take another example, Elster considers whether norms exist to promote self-interest (or common interest or genetic fitness).

The work on social norms that derives from the investigations of Sen circumvents some of the aforementioned philosophical problems by taking social norms for granted and representing them in terms of constraints on feasibility. We followed Sen here, utilizing a permissibility operator $\pi$ that when applied to a menu of options returns those alternatives which are permitted for choice by the underlying norm. We have not imposed substantial constraints on the permissibility operator. Some of the questions which philosophers ask about norms can be reconstructed in terms of additional constraints on $\pi$. We leave this for future work, and we follow the tradition inaugurated by Sen and continued by Bossert and Suzumura, which uses a fairly unconstrained permissibility operator.

We considered the natural idea about how to extend the notion of rationalizability to cases where underlying norms influence choice. In this context, we called a choice function $\gamma \pi$-rationalizable if there is a binary relation $R$ such that:

$$
\gamma(S)=G(\pi(S), R):=\{x \in \pi(S): x R y \text { for all } y \in \pi(S)\} .
$$

The challenge then was to characterize functionally this extended notion of rationalizabillity. The work done on rationalizability in Section 1.2 is helpful here. In fact, there is a natural variant of condition $\gamma \mathbf{R}_{\infty}$ that does the job (viz., condition $\gamma[\pi] \mathbf{R}_{\infty}$ ). The functional characterization holds with respect to general domains.

Bossert and Suzumura also offer in [BS07] a characterization of this extended notion of rationalizability. In fact, their characterization also holds for general domains, but the condition that they propose implements a notion of revealed preference in its formulation. 
This type of formulation seems less direct than a condition formulated purely in terms of the choice operator, such as our $\gamma[\pi] \mathbf{R}_{\infty}$. Indeed, our condition has proven to be useful for the epistemological application we discussed in the second part of this article (while Bossert and Suzumura's condition seems difficult to apply in this context).

The second half of this article applied the results of the first part to epistemology. First we offered some examples showing that social norms also play a significant role in undermining crucial principles of belief revision which guarantee that belief revision functions are relational. Rott has offered examples of this kind in [Rot04], although these examples do not turn on the role of social norms but on a more general phenomenon that Sen calls menu dependence. In any case, Rott offer examples but he does propose a solution. To be sure, he writes, "We have identified a formidable problem, but we havent been able to offer an acceptable solution for it. But problems there are, and creating awareness of problems is one of the important tasks of philosophy" [Rot04, p. 238].

One of the main goals of the second half of this article was to offer a solution to the sort of counterexamples we presented. Our solution can be extended to cover counterexamples of the sort offered by Rott; we intend to deal with the general issue of menu dependence in a companion article. The idea behind our solution is to use the generalized notion of rationalizability in the area of belief change. Thus, we have here as well a permissibility function $\pi$ and the usual selection function $\gamma$ familiar to students of belief change since its use in [AGM85]. Yet corresponding to these mechanisms are two components which comprise belief change, 困 and $*$, which can be defined in terms of the corresponding selection functions:

$$
K * \varphi:= \begin{cases}\widehat{\gamma(\llbracket \varphi \rrbracket)} & \text { if } \gamma(\llbracket \varphi \rrbracket) \neq \emptyset \\ \operatorname{For}(\mathcal{L}) & \text { otherwise }\end{cases}
$$

and

$$
K \circledast \varphi:= \begin{cases}\widehat{\pi(\llbracket \varphi \rrbracket)} & \text { if } \pi(\llbracket \varphi \rrbracket) \neq \emptyset \\ \operatorname{For}(\mathcal{L}) & \text { otherwise. }\end{cases}
$$

We verify that when the language is finite, condition $\gamma[\pi] \mathbf{R}_{\infty}$ is mappable to a novel mixed postulate combining the $*$ and $⿴$ operators (viz., postulate $*\left[\right.$ 困] $\mathrm{R}_{\infty}$ ). This yields an extension of the constraint that belief revision is relational to the case where social norms are operative. Finally we offer a complete characterization for the two change operators.

The mappings linking selection functions and belief revision operators provide heuristic insight about the shape of desired postulates in the area of belief change. It seems that the same method can be used to deal with the general issue of menu dependence, although this phenomenon remains a bit more elusive. Social norms offer a mechanism that explains shifts in feasibility, yet other cases of menu dependence involve shifts which are purely epistemic and more difficult to explain. Nonetheless, perhaps by interpreting the underlying formalism in a different way, the theory offered here can be lent a broader interpretation that covers other cases of menu dependence.

The general moral to be taken from this article and from recent work by Bossert and Suzumura is that the theory of rational choice can be extended fruitfully in order to cope with counterexamples involving social norms. By the same token, the origin of the counterexamples to principles of belief formation offered by Rott in [Rot04] resides in the same phenomenon that motivated Sen's counterexamples against fundamental conditions in the theory of choice. As we have seen, social norms can be a source of counterexamples to 
principles of belief formation. A solution to problems in the theory of choice inspired a solution in the realm of belief change.

\section{REFERENCES}

[AGM85] Carlos E. Alchourrón, Peter Gärdenfors, and David Makinson, On the Logic of Theory Change: Partial Meet Contraction and Revision Functions, The Journal of Symbolic Logic 50 (1985), no. 2, 510 530.

[AM85] Carlos E. Alchourrón and David Makinson, On the Logic of Theory Change: Safe Contraction, Studia Logica 44 (1985), no. 4, 405-422.

[Ar195] Horacio Arló-Costa, Epistemic Conditionals, Snakes and Stars, Conditionals: from Philosophy to Computer Science (A. Herzig L. Farinas del Cerro, G. Crocco, ed.), Studies in Logic and Computation, vol. 5, Oxford University Press, 1995, pp. 193-239.

[Ar106] - Rationality and Value: The Epistemological Role of Interdeterminate and Agent-Dependent Values, Philosophical Studies 128 (2006), no. 1, 7-48

[Arr51] Kenneth J. Arrow, Social choice and individual values, 1 ed., Wiley, 1951.

[Arr59]_, Rational Choice Functions and Orderings, Economica 26 (1959), 121-127.

[Bic06] Cristina Bicchieri, The Grammar of Society: The Nature and Dynamics of Social Norms, Cambridge University Press, 2006.

[BS07] W. Bossert and K. Suzumura, Social Norms and Rationality of Choice, Preprint, Département de Sciences Economiques, Université de Montréal, 2007, August 2007.

[Els89a] Jon Elster, Nuts and bolts for the social sciences, Cambridge University Press, 1989.

[Els89b] _ Social Norms and Economic Theory, The Journal of Economic Perspectives 3 (1989), no. 4, 99-117.

[Gär79] Peter Gärdenfors, Conditionals and Changes of Belief, Logic and Epistemology of Scientific Change (I Niiniluoto and R. Tuomela, eds.), Acta Philosophica Fennica, vol. 30, North-Holland Publishing Company, 1979, pp. 381-404.

[Gär88] _ Knowledge in flux. modeling the dynamics of epistemic states, The MIT Press, 1988.

[Gär92] _ Belief Revision: An Introduction, Belief Revision (Peter Gärdenfors, ed.), Cambridge University Press, 1992, pp. 1-28.

[GM94] Peter Gärdenfors and David Makinson, Nonmonotonic Inference Based on Expectations, Artificial Intelligence 65 (1994), no. 2, 197-245.

[GR95] Peter Gärdenfors and Hans Rott, Belief Revision, Handbook of Logic in Artificial Intelligence and Logic Programming (Dov M. Gabbay, C.J. Hogger, and J.A. Robinson, eds.), vol. 4, Oxford University Press, 1995, pp. 35-132.

[Gro88] Adam Grove, Two Modellings for Theory Change, Journal of Philosophical Logic 17 (1988), no. 2, $157-170$

[Han68] B. Hansson, Choice Structures and Preference Relations, Synthese 18 (1968), no. 4, 443-458.

[Han99] Sven Ove Hansson, A textbook of belief dynamics: Theory change and database updating, Kluwer Academic Publishers, 1999.

[Her73] Hans G. Herzberger, Ordinal Preference and Rational Choice, Econometrica 41 (1973), no. 2, 187-237.

[KLM90] Sarit Kraus, Daniel Lehmann, and Menachem Magidor, Nonmonotonic Reasoning, Preferential Models and Cumulative Logics, Artificial Intelligence 44 (1990), no. 1-2, 167-207.

[Lev96] Isaac Levi, For the Sake of the Argument, Cambridge University Press, 1996.

[Lev04a] _ Inclusive Rationality: A Review of Amartya Sen: Rationality and Freedom, The Journal of Philosophy 101 (2004), no. 5, 255-276.

[Lev04b] _ Mild Contraction, Oxford University Press, 2004.

[LM92] Daniel Lehmann and Menachem Magidor, What Does a Conditional Knowledge Base Entail?, Artificial Intelligence 55 (1992), no. 1, 1-60.

[Mak89] David Makinson, General Theory of Cumulative Inference, Proceedings of the 2nd International Workshop on Non-Monotonic Reasoning, Lecture Notes in Computer Science, vol. 346, Springer-Verlag, 1989, pp. 1-18.

[MG91] David Makinson and Peter Gärdenfors, Relations between the Logic of Theory Change and Nonmonotonic Logic, The Logic of Theory Change (André Fuhrmann and Michael Morreau, eds.), Lecture Notes in Computer Science, Springer-Verlag, 1991, pp. 185-205.

[Mou85] H. Moulin, Choice Functions over a Finite Set: A Summary, Social Choice and Welfare 2 (1985), $147-160$ 
[Ols03] Erik J. Olsson, Belief Revision, Rational Choice and the Unity of Reason, Studia Logica 73 (2003), no. 2, 219-240.

[Ped08] Arthur Paul Pedersen, Rational Choice and Belief Revision: An Essay in Formal Epistemology, Master's thesis, Carnegie Mellon University, Department of Philosophy, 2008.

[PG96] Judea Pearl and M. Goldszmidt, Qualitative Probabilities for Default Reasoning, Belief Revision, and Causal Modeling, Artificial Intelligence 84 (1996), no. 1-2, 57-112.

[Ray73] Paramesh Ray, Independence of Irrelevant Alternatives, Econometrica 41 (1973), no. 5, 987-991.

[Ric66] M.K. Richter, Revealed Preference Theory, Econometrica 34 (1966), no. 3, 635-645.

[Ric71] _ Rational Choice, Preferences, Utility, and Demand (J. Chipman, L. Hurwicz, M. Richter, and H. Sonnenshein, eds.), Harcourt Brace Javanovich, 1971, pp. 29-58.

[Rot93] Hans Rott, Belief Contraction in the Context of the General Theory of Rational Choice, Journal of Symbolic Logic 58 (1993), no. 4, 1426-1450.

[Rot01] Change, choice and inference: A study of belief revision and nonmonotonic reasoning, Oxford University Press, 2001.

[Rot04] _ A Counterexample to Six Fundamental Principles of Belief Formation, Synthese 139 (2004), no. $2,225-240$.

[RP00] Hans Rott and Maurice Pagnucco, Severe Withdrawal (and Recovery), Journal of Philosophical Logic 28 (2000), no. 5, 501-547.

[Sam38] Paul A. Samuelson, A Note on the Pure Theory of Consumers, Economica 5 (1938), 61-71.

[Sam47] , Foundations of economic analysis, Harvard University Press, 1947.

[Sen70] Amartya Sen, Collective choice and social welfare, Holden-Day, 1970.

[Sen71] _ Choice Functions and Revealed Preference, Review of Economic Studies 38 (1971), 307-317.

[Sen77] _ Social Choice Theory: A Re-Examination, Econometrica 45 (1977), no. 1, 53-89.

[Sen93] _ Internal Consistency of Choice, Econometrica 61 (1993), no. 3, 495-521.

[Sen96] Is the Idea of Purely Internal Consistency of Choice Bizarre?, Language, World and Reality (J.E.J Altham and T.R. Harrison, eds.), A Festschrift for Bernard Williams, Cambridge University Press, 1996, pp. 19-31.

[Sen97] _ Maximization and the Act of Choice, Econometrica 65 (1997), no. 4, 745-779.

[Spo] Wolfgang Spohn, A Survey in Ranking Theory, To appear in F. Huber, C. Schmidt-Petri, editors, Degrees of Belief. An Anthology, Oxford University Press, Oxford, 52 pages.

[Suz76] Kotaro Suzumura, Rational Choice and Revealed Preference, Review of Economic Studies 43 (1976), no. $1,149-158$.

[Suz83] _ Rational choice, collective decisions, and social welfare, Cambridge University Press, 1983. 